\title{
Random matrix approach to three-dimensional QCD with a Chern-Simons term
}

\author{
Takuya Kanazawa, ${ }^{a}$ Mario Kieburg ${ }^{b}$ and Jacobus J.M. Verbaarschot ${ }^{c}$ \\ ${ }^{a}$ Research and Development Group, Hitachi, Ltd., \\ Kokubunji, Tokyo 185-8601, Japan \\ ${ }^{b}$ School of Mathematics and Statistics, University of Melbourne, \\ Parkville, Melbourne VIC 3010, Australia \\ ${ }^{c}$ Department of Physics and Astronomy, Stony Brook University, \\ Stony Brook, NY 11794, U.S.A. \\ E-mail: asuka12477@gmail.com, m.kieburg@unimelb.edu.au, \\ jacobus. verbaarschot@stonybrook. edu
}

ABSTRACT: We propose a random matrix theory for QCD in three dimensions with a Chern-Simons term at level $k$ which spontaneously breaks the flavor symmetry according to $\mathrm{U}\left(2 N_{\mathrm{f}}\right) \rightarrow \mathrm{U}\left(N_{\mathrm{f}}+k\right) \times \mathrm{U}\left(N_{\mathrm{f}}-k\right)$. This random matrix model is obtained by adding a complex part to the action for the $k=0$ random matrix model. We derive the pattern of spontaneous symmetry breaking from the analytical solution of the model. Additionally, we obtain explicit analytical results for the spectral density and the spectral correlation functions for the Dirac operator at finite matrix dimension, that become complex. In the microscopic domain where the matrix size tends to infinity, they are expected to be universal, and give an exact analytical prediction to the spectral properties of the Dirac operator in the presence of a Chern-Simons term. Here, we calculate the microscopic spectral density. It shows exponentially large (complex) oscillations which cancel the phase of the $k=0$ theory.

Keywords: Matrix Models, Chern-Simons Theories, Chiral Lagrangians, Field Theories in Lower Dimensions

ARXiV EPrint: 1904.03274 


\section{Contents}

1 Introduction 1

2 Random matrix model 3

2.1 Partition function of our model 3

2.2 Chern-Simons term and $\eta$-invariant 5

2.3 Derivation of the sigma model at large $N \quad 7$

2.4 Phase transitions at non-integer $k \quad 9$

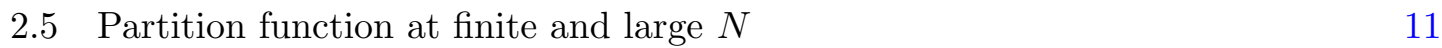

3 Spectral correlation functions $\quad \mathbf{1 5}$

$\begin{array}{lll}3.1 & \text { General } N_{\mathrm{f}} \text { at finite } N & 16\end{array}$

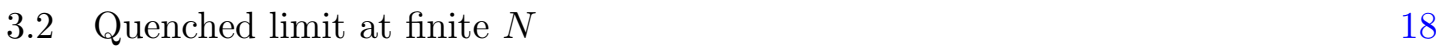

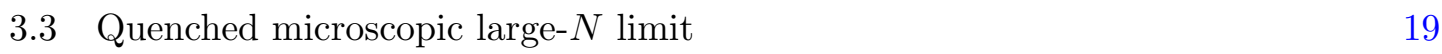

3.4 Unquenched microscopic level density 23

4 Conclusion and outlook 28

A Derivation of some partition functions $\quad 29$

A.1 Quenched $A$ integral $\quad 29$

$\begin{array}{ll}\text { A.2 } & \text { Unquenched } A \text { integral }\end{array}$

A.3 The $B$ integral 33

\section{Introduction}

It is believed that in the QCD vacuum the strong interactions of gluons and quarks induce spontaneous breaking of chiral symmetry $\mathrm{SU}\left(N_{\mathrm{f}}\right)_{R} \times \mathrm{SU}\left(N_{\mathrm{f}}\right)_{L} \rightarrow \mathrm{SU}\left(N_{\mathrm{f}}\right)_{V}$ when the number of massless Dirac fermions $N_{\mathrm{f}}$ is below the conformal window. The quantum fluctuations of the gauge fields in the broken phase manifest themselves in characteristic spectral fluctuations of the Dirac operator in the microscopic domain [1], which can be exactly reproduced by a zero-dimensional matrix model with the same global symmetries as QCD known as chiral random matrix theory [2-4]. We refer to [5-9] for reviews.

In three-dimensional spacetime, whether the symmetry of fermions is dynamically broken or not has remained a matter of debate for decades [10-12]. Three-dimensional gauge theories are of distinguished importance in various contexts, ranging from domain walls and surface (boundary) states in four dimensions to quantum Hall effects, graphene, spin liquids and high-temperature superconductivity [13-17]. Part of this rich physics stems from the existence of a Chern-Simons term. In three-dimensional QED $\left(\mathrm{QED}_{3}\right)$ the interplay of a Chern-Simons term and fermionic symmetry breaking was investigated 
in [18-24]. A consensus from these studies is that dynamical symmetry breaking is generally suppressed by a Chern-Simons term, because photons acquire a gauge-invariant mass term which in turn quenches quantum fluctuations. However, recent lattice simulations [25-27] report that dynamical mass generation of fermions does not occur in $\mathrm{QED}_{3}$ with two twocomponent Dirac fermions even in the absence of a Chern-Simons term (see also [28, 29] for studies on $\mathrm{QED}_{3}$ with a single Dirac fermion).

In contrast, in three-dimensional QCD $\left(\mathrm{QCD}_{3}\right)$ with an even number $2 N_{\mathrm{f}}$ of massless two-component flavors and without a Chern-Simons term, dynamical symmetry breaking ${ }^{1}$

$$
\mathrm{U}\left(2 N_{\mathrm{f}}\right) \rightarrow \mathrm{U}\left(N_{\mathrm{f}}\right) \times \mathrm{U}\left(N_{\mathrm{f}}\right)
$$

is believed to take place through fermion bilinear condensation when $N_{\mathrm{f}}$ is below a certain threshold [31-33]. The fermion condensate has been observed in quenched lattice simulations $[34,35]$. A non-chiral matrix model corresponding to (1.1) is also known [36]; see [37-46] for further developments. However, not much is known about $\mathrm{QCD}_{3}$ at nonzero Chern-Simons level $k .^{2}$ This is partly due to the sign problem that makes a direct lattice simulation prohibitively hard. Recently, it was argued [48] that there is a finite window of $N_{\mathrm{f}}$ in which a novel symmetry breaking

$$
\mathrm{U}\left(2 N_{\mathrm{f}}\right) \rightarrow \mathrm{U}\left(N_{\mathrm{f}}+k\right) \times \mathrm{U}\left(N_{\mathrm{f}}-k\right) \quad \text { for } \quad|k|<N_{\mathrm{f}}
$$

occurs. New boson-fermion dualities describing the transition region of (1.2) were also proposed [48]. While a proof is not available yet, this conjecture passes nontrivial tests such as the matching of symmetries and anomalies, and consistency under mass deformations. Related discussions can be found in [49, 50].

In this paper, we propose a new random matrix model that realizes the symmetry breaking scenario (1.2). ${ }^{3}$ This is made possible through a judicious choice of a non-Gaussian weight for matrix elements in which $k$ enters as a parameter. We show that in the large- $N$ limit with $N$ the matrix size, the model reduces to a sigma model with a target space of the complex Grassmannian $\mathrm{U}\left(2 N_{\mathrm{f}}\right) /\left[\mathrm{U}\left(N_{\mathrm{f}}+k\right) \times \mathrm{U}\left(N_{\mathrm{f}}-k\right)\right]$. When $k$ is varied there occurs a sequence of first order phase transitions that separate phases with different complex Grassmannians. By solving the model we delineate the structure of the Dirac operator spectrum that underlies the exotic symmetry breaking (1.2). Under the assumption that (1.2) indeed characterizes the vacuum of $\mathrm{QCD}_{3}$ with a Chern-Simons term, our approach offers an entirely new way to probe the interplay of strongly coupled fermion dynamics and a topological term within a tractable framework.

This paper is organized as follows. In section 2 we define the model and derive key properties. The partition function of the model is computed and the phase structure as a function of $k$ and the fermion mass is investigated. Section 3 is devoted to the

\footnotetext{
${ }^{1}$ A Vafa-Witten-type argument [30] shows that the $\mathrm{U}\left(N_{\mathrm{f}}\right) \times \mathrm{U}\left(N_{\mathrm{f}}\right)$ symmetry is unbroken. Here we assume that fermions are in a complex representation of the gauge group.

${ }^{2}$ As is well known, non-Abelian gauge invariance forces the Chern-Simons coefficient to be quantized [47]. Here we label it as $k \in \mathbb{Z}$.

${ }^{3}$ To avoid confusion we note that the approach of the present paper is unrelated to Chern-Simons matrix models in [51-55], where the dynamics of fermions was not the main focus.
} 
spectral functions that are first derived at finite matrix dimension $N$. As $k$ grows the spectral density evolves from a smooth semicircle to a distorted complex oscillatory form. In addition, we compute the large- $N$ microscopic limit of the spectral density in the quenched and unquenched ensemble. The details of the calculations to get these results are given in appendix A. Concluding remarks are made in section 4 .

\section{Random matrix model}

We introduce three new random matrix models labeled by the Dyson index $\beta$ in subsection 2.1. They are associated with $\mathrm{QCD}_{3}$ in the presence of a Chern-Simons term with fermions transforming in a complex/pseudoreal/real $(\beta=2 / 1 / 4)$ representation of the gauge group, respectively. The class $\beta=2$ comprises quarks in the fundamental representation of $\mathrm{SU}\left(N_{c}\right)$ with $N_{c} \geq 3 ; \beta=1$ includes quarks in the fundamental representation of $\operatorname{USp}\left(N_{c}\right)$ (here, $N_{c}$ must be even) $;{ }^{4}$ and $\beta=4$ corresponds to quarks in the adjoint representation of $\mathrm{SU}\left(N_{c}\right)^{5}$ and in the vector representation of $\mathrm{SO}\left(N_{c}\right)$. In subsection 2.2 we give a discussion on the renormalization of the Chern-Simons term due to the dynamical quarks that are related to the $\eta$-invariant.

Each of the above three random matrix models produces a universal non-linear sigma model that is derived in detail for $\beta=2$ in subsection 2.3. As in the case of three dimensional QCD, the model experiences a phase transition from one to another sigma model due to the Chern-Simons-like term. In subsection 2.4 we show how the mechanism works in general, and in subsection 2.5 we illustrate our findings by studying the two-flavor case for $\beta=2$. Therein, we also present finite results for the partition function at finite matrix dimension.

\subsection{Partition function of our model}

The partition functions of our model are defined by

$$
\begin{aligned}
Z^{\beta=2} & =\int \mathrm{d} A \exp \left[\frac{\alpha_{2}}{2}(\operatorname{Tr} A-2 i k)^{2}-\frac{N}{2} \operatorname{Tr} A^{2}\right] \prod_{f=1}^{2 N_{\mathrm{f}}} \operatorname{det}\left(i A+m_{f} \mathbb{1}_{N}\right) \\
Z^{\beta=1} & =\int \mathrm{d} A^{\prime} \exp \left[\alpha_{1}\left(\operatorname{Tr} A^{\prime}-2 i k\right)^{2}-N \operatorname{Tr} A^{\prime 2}\right] \prod_{f=1}^{2 N_{\mathrm{f}}} \operatorname{det}\left(i A^{\prime}+m_{f} \mathbb{1}_{N}\right) \\
Z^{\beta=4} & =\int \mathrm{d} A^{\prime \prime} \exp \left[\frac{\alpha_{4}}{4}\left(\operatorname{Tr} A^{\prime \prime}-2 i k\right)^{2}-\frac{N}{2} \operatorname{Tr} A^{\prime \prime 2}\right] \prod_{f=1}^{2 N_{\mathrm{f}}} \sqrt{\operatorname{det}\left(i A^{\prime \prime}+m_{f} \mathbb{1}_{2 N}\right)}
\end{aligned}
$$

where $A$ is a complex hermitian $N \times N$ matrix, $A^{\prime}$ is a real symmetric $N \times N$ matrix, and $A^{\prime \prime}$ is a self-dual $N \times N$ matrix whose elements are real quaternions. ${ }^{6}$ The measures $\mathrm{d} A$,

\footnotetext{
${ }^{4}$ Our convention is such that $\mathrm{USp}(2)=\mathrm{SU}(2)$.

${ }^{5}$ There is an argument against spontaneous symmetry breaking in large- $N_{c}$ QCD 3 with adjoint fermions [56].

${ }^{6}$ The matrix size $N$ is not equal to the number of colors in $\mathrm{QCD}_{3}$. It replaces the dimension of the Hilbert space that is the product of the space-time volume, number of colors and spinor dimension (dimension of the gauge group representation).
} 
$\mathrm{d} A^{\prime}$ and $\mathrm{d} A^{\prime \prime}$ are the corresponding Lebesgue measures, in particular the products of the differentials of the real independent matrix entries. The square root of the determinants for $\beta=4$, see (2.3), is exact and may be implemented as a Pfaffian determinant,

$$
\sqrt{\operatorname{det}\left(i A^{\prime \prime}+m_{f} \mathbb{1}_{2 N}\right)}=\operatorname{Pf}\left[\left(-i \sigma_{2} \otimes \mathbb{1}_{N}\right)\left(i A^{\prime \prime}+m_{f} \mathbb{1}_{2 N}\right)\right]
$$

The masses are gathered in the diagonal matrix $M=\operatorname{diag}\left(m_{1}, \ldots, m_{2 N_{\mathrm{f}}}\right)$.

The positive constant $\alpha_{\beta}$ determines the effective strength of the "Chern-Simons" coupling. Starting with section 2.5 it will be chosen

$$
\alpha_{\beta}=\frac{N}{N+4 N_{\mathrm{f}} / \beta+1}<1
$$

In sections 2.3 and 2.4 the detailed form of $\alpha_{\beta}$ is irrelevant apart from the convergence requirements of the integrals and, thence, remain unspecified therein. Indeed the integrability of the variable $\operatorname{Tr} A$ is guaranteed when $\alpha_{1}<1, \alpha_{2}<1$ and $\alpha_{4}<2$, which is satisfied by eq. (2.5).

In eq. (2.3) $A^{\prime \prime}$ is regarded as a complex $2 N \times 2 N$ matrix, using $\left(\mathbb{1}_{2}, i \sigma_{a}\right)$ as the quaternion basis. Note that all matrices are square, reflecting the absence of topological zero modes in $2+1$ dimensions. The real parameter $k$ corresponds to the Chern-Simons level, and $2 N_{\mathrm{f}}$ represents the number of two-component Dirac fermions for $\beta=1,2$ and of two-component Majorana fermions for $\beta=4$. The case of an odd number of flavors will not be considered in this paper. We expect that the mechanism described below should work similar to the even number of flavors case though their is a significant difference; the Goldstone manifold is disconnected for an odd number of flavors [36, 41, 42].

The models (2.1), (2.2) and (2.3) differ from the conventional random matrix models for $\mathrm{QCD}_{3}[36,41,42]$ by the presence of the squared trace term in the exponent. ${ }^{7}$ At $k \neq 0$, the latter makes the statistical weight complex-valued, just as the Chern-Simons term does in Euclidean $\mathrm{QCD}_{3}$ causing the infamous "sign problem". It is not problematic for us because we can still solve the matrix models exactly without recourse to numerical simulations. Our motivation to include a squared trace term is that this deformation changes the pattern of flavor symmetry breaking. The microscopic large- $N$ limit $[2,3]$ makes this more lucid. For this purpose, we take $N \rightarrow \infty$ and $m_{f} \rightarrow 0$ with $\widehat{m}_{f}=N m_{f}$ and $k$ fixed. ${ }^{8}$ If $N$ is identified with the volume of space-time, this limit is equivalent to the leading order of the $\varepsilon$-expansion in chiral perturbation theory [1, 64], in which the partition function reduces to a non-linear sigma model of static Nambu-Goldstone modes. If $k \in \mathbb{Z}$ with $|k| \leq N_{\mathrm{f}}$, one can show for the partition functions (2.1), (2.2) and (2.3) in

\footnotetext{
${ }^{7}$ A squared trace term was first also introduced in matrix models in [57] with application to 2D quantum gravity, see also [58-62]. Additionally, they appear in random matrix theories for the Wilson Dirac operator [63].

${ }^{8}$ This limit should not be confused with the large- $N_{c}$ limit in gauge theory. We keep the number of colors $N_{c}$ in $\mathrm{QCD}_{3}$ finite throughout our work.
} 
the microscopic limit reduce to

$$
\begin{aligned}
Z^{\beta=2} & \sim \int_{\mathrm{U}\left(2 N_{\mathrm{f}}\right)} \mathrm{d} \mu(U) \exp \left[\operatorname{Tr} U^{\dagger} \Lambda_{k} U \widehat{M}\right], \\
Z^{\beta=1} & \sim \int_{\mathrm{USp}\left(4 N_{\mathrm{f}}\right)} \mathrm{d} \mu(U) \exp \left[\operatorname{Tr} U^{\dagger} \operatorname{diag}\left(\Lambda_{k},-\Lambda_{k}\right) U \operatorname{diag}(\widehat{M},-\widehat{M})\right], \\
Z^{\beta=4} & \sim \int_{\mathrm{O}\left(2 N_{\mathrm{f}}\right)} \mathrm{d} \mu(O) \exp \left[\operatorname{Tr} O^{\mathrm{T}} \Lambda_{k} O \widehat{M}\right]
\end{aligned}
$$

where $\widehat{M} \equiv \operatorname{diag}\left(\widehat{m}_{1}, \ldots, \widehat{m}_{2 N_{\mathrm{f}}}\right)$ and

$$
\Lambda_{k} \equiv \operatorname{diag}\left(\mathbb{1}_{N_{\mathrm{f}}+k},-\mathbb{1}_{N_{\mathrm{f}}-k}\right) .
$$

The Haar measure of the respective groups are denoted by $\mathrm{d} \mu$. This result is derived in subsection 2.3 .

Effectively we do not integrate over the whole group but a coset. These cosets are the Goldstone manifolds and reflect the patterns of flavor symmetry breaking in the "chiral" limit given by

$$
\begin{aligned}
\beta & =2: & \mathrm{U}\left(2 N_{\mathrm{f}}\right) & \rightarrow \mathrm{U}\left(N_{\mathrm{f}}+k\right) \times \mathrm{U}\left(N_{\mathrm{f}}-k\right), \\
\beta & =1: & \mathrm{USp}\left(4 N_{\mathrm{f}}\right) & \rightarrow \mathrm{USp}\left(2\left(N_{\mathrm{f}}+k\right)\right) \times \mathrm{USp}\left(2\left(N_{\mathrm{f}}-k\right)\right), \\
\beta & =4: & \mathrm{O}\left(2 N_{\mathrm{f}}\right) & \rightarrow \mathrm{O}\left(N_{\mathrm{f}}+k\right) \times \mathrm{O}\left(N_{\mathrm{f}}-k\right)
\end{aligned}
$$

yielding $2\left(N_{\mathrm{f}}^{2}-k^{2}\right), 4\left(N_{\mathrm{f}}^{2}-k^{2}\right)$ and $N_{\mathrm{f}}^{2}-k^{2}$ Nambu-Goldstone modes, respectively. When $k=0$, we regain the usual symmetry breaking patterns proposed for parity-invariant $\mathrm{QED}_{3}$ and $\mathrm{QCD}_{3}$ with no Chern-Simons term $[10,12,31,36,41,42,44,46]$. This agreement is nontrivial because the partition functions (2.1), (2.2) and (2.3) are different from those in $[36,41,42]$ even at $k=0$ due to the squared trace term. It highlights the universality of the large- $N$ limit. When $k \neq 0$, the symmetry breaking schemes (2.10) coincide with the generalizations proposed recently [48] for $\mathrm{QCD}_{3}$ with a Chern-Simons term at level $k$.

In (2.6), (2.7) and (2.8) we omitted overall multiplicative factors, which are all proportional to $(-1)^{N k}$. Therefore choosing even $N$ is mandatory to ensure positivity of the partition function, although the overall normalization of $Z$ does not affect physical expectation values.

\subsection{Chern-Simons term and $\eta$-invariant}

The parameter $k$ is not the only source of the Chern-Simons-level as we will show for the Dyson index $\beta=2$. For $M \rightarrow 0$ the phase of the fermion determinant also contributes by the $\eta$ invariant $\eta(A)=\sum_{j=1}^{N} \operatorname{sign}\left(\lambda_{j}\right)$, see [65-68], as follows

$$
\prod_{f=1}^{2 N_{\mathrm{f}}} \operatorname{det}\left(i A+m_{f} \mathbb{1}_{N}\right) \stackrel{|M| \rightarrow 0}{\approx}|\operatorname{det} A|^{2 N_{\mathrm{f}}} e^{i \pi N_{\mathrm{f}} \sum_{j=1}^{N} \operatorname{sign}\left(\lambda_{j}\right)} .
$$




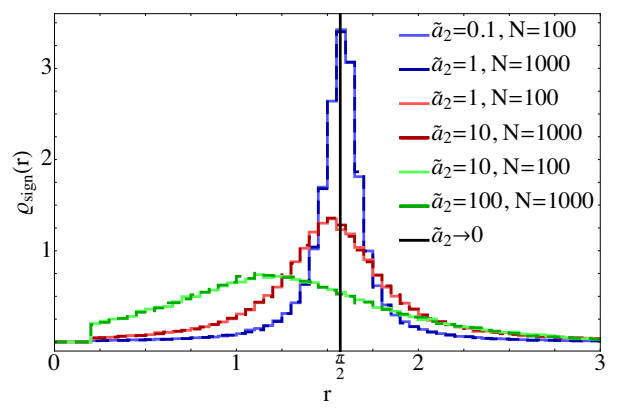

Figure 1. Distribution $\rho_{\text {sign }}(r)$ of the ratio $r=\operatorname{Tr} A / \operatorname{Tr} \operatorname{sign}(A)$ for $k=0$ and $N_{\mathrm{f}}=0$ generated by Monte Carlo simulations. The matrix size $N$ is chosen to be one of the two values $N=100$ (bright solid histograms) and $N=1000$ (dark dashed histograms) and the parameter $\alpha_{2}=1-\widetilde{a}_{2} / N$ takes three values with the ratios $\widetilde{a}_{2} / N=10^{-1}$ (green), $\widetilde{a}_{2} / N=10^{-2}$ (red), and $\widetilde{a}_{2} / N=10^{-3}$ (blue). The ensemble size varies because we omitted those configurations with $r=\infty$, i.e. $\operatorname{Tr} \operatorname{sign}(A)=0$. The number of these configurations decreases according to $\sqrt{\widetilde{a}_{2} / N}$. The limit $\widetilde{a}_{2} / N \rightarrow 0$ (the physical case) becomes a Dirac delta distribution at $r=\pi / 2$ (black vertical line). Since the histograms with fixed quotient $\widetilde{a}_{2} / N$ agree almost perfectly, they are barely distinguishable, we are save to assume that the plots show the limiting large $N$ behavior.

This can be combined with the imaginary part of the first term in the exponent of eq. (2.1), which can be written as

$$
-2 i k \alpha_{2} \operatorname{Tr} A \stackrel{N \gg 1,\left|1-\alpha_{2}\right| \ll 1}{\approx}-\pi i k \sum_{j=1}^{N} \operatorname{sign}\left(\lambda_{j}\right) .
$$

This approximation can be seen by considering the integral (2.1) for $k=0$ which gives a quenched approximation for $\operatorname{Tr} A$. The integral can be rewritten as

$$
Z^{\beta=2}=c \int \mathrm{d} A \int \mathrm{d} x \mathrm{e}^{-\frac{\alpha_{2}\left(1-\alpha_{2}\right)}{2} x^{2}-\frac{N}{2} \operatorname{Tr}(A-\alpha x / N)^{2}} .
$$

For the physically interesting limit of $\alpha=1-O(1 / N)$, we have that $x \sim O(\sqrt{N})$, so that $\operatorname{Tr} A$ fluctuates with a magnitude of $O(1 / \sqrt{N})$, which is much larger than the average level spacing of $O(1 / N)$. These fluctuations are collective, meaning that all eigenvalues of $A$ move up and down with $x$ in the same way. Therefore, starting with a configuration with an equal number of eigenvalues on the left and right of zero, a fluctuation where $k$ eigenvalues move to the right changes $\operatorname{Tr} A$ by

$$
\delta \operatorname{Tr} A=N k \Delta \lambda
$$

and $\Delta \lambda=\pi / N$ the level spacing so that

$$
N \Delta \lambda k=\pi k,
$$

The sum of the sign of the eigenvalues changes by

$$
\delta \sum_{i} \operatorname{sign} \lambda_{i}=2 k
$$


This results in the ratio

$$
\frac{\sum_{i} \lambda_{i}}{\sum_{i} \operatorname{sign} \lambda_{i}}=\frac{\sum_{i} \delta \lambda_{i}}{\sum_{i} \delta \operatorname{sign} \lambda_{i}}=\frac{\pi}{2}
$$

which is the desired relation (2.12). This relation (2.12) has also been checked numerically, see figure 1, where the distribution of the ratio $r=\sum_{j=1}^{N} \lambda_{j} / \sum_{j=1}^{N} \operatorname{sign}\left(\lambda_{j}\right)$ has been generated with Monte Carlo simulations.

Summarizing, the phase of the fermion determinant renormalizes the bare ChernSimons level as

$$
-\pi i k_{\mathrm{rn}} \eta(A)=-\pi i\left(k-N_{\mathrm{f}}\right) \eta(A) .
$$

Therefore, the action occurs as in three-dimensional QCD, see eq. (1.4) in [48]. ${ }^{9}$ We will see in the ensuing discussion that the pattern of chiral symmetry breaking does not depend on $\alpha_{\beta}$, as long as the integrals are convergent, which is certainly the case for any value of $\alpha_{\beta}<N /\left(N+4 N_{\mathrm{f}} / \beta\right)<1$. The slightly smaller bound than 1 avoids that the integral over $\operatorname{Tr} A$ does not diverge (this can be seen after splitting $A$ into its trace and a traceless part).

\subsection{Derivation of the sigma model at large $N$}

The derivations of the partitions functions (2.6), (2.7) and (2.8) are similar, and we, therefore, outline only the $\beta=2$ class here. The procedure follows standard steps [2,69]. First we linearize the squared trace term at the expense of a new Gaussian integral over an auxiliary variable $x$ and, afterwards, shift $A \rightarrow A-x \mathbb{1}_{N}$ to eliminate the linear term in $A$. This makes it clear that the partition function of our model is nothing but a reweighted integral of the ordinary Gaussian matrix model ${ }^{10}$

$$
\begin{aligned}
Z^{\beta=2} & =\frac{N}{\sqrt{2 \pi \alpha_{2}}} \int_{-\infty}^{\infty} \mathrm{d} x \exp \left[-\frac{N^{2}}{2} \frac{1-\alpha_{2}}{\alpha_{2}} x^{2}+2 i N k x\right] \mathcal{Z}_{N, N_{\mathrm{f}}}(M-i x), \\
\mathcal{Z}_{N, N_{\mathrm{f}}}(M-i x) & \equiv \int \mathrm{d} A \exp \left[-\frac{N}{2} \operatorname{Tr} A^{2}\right] \prod_{f=1}^{2 N_{\mathrm{f}}} \operatorname{det}\left[i A+\left(m_{f}-i x\right) \mathbb{1}_{N}\right] .
\end{aligned}
$$

Upon rewriting the determinant in terms of Grassmann variables,

$$
\operatorname{det}\left[i \widetilde{A}+\left(m_{f}-i x\right) \mathbb{1}_{N}\right]=\frac{\int \mathrm{d} \psi_{f} \mathrm{~d} \bar{\psi}_{f} \exp \left[-\sum_{a, b=1}^{N} \bar{\psi}_{f}^{a}\left(i \widetilde{A}+\left(m_{f}-i x\right) \mathbb{1}_{N}\right)_{a b} \psi_{f}^{b}\right]}{\int \mathrm{d} \psi_{f} \mathrm{~d} \bar{\psi}_{f} \exp \left[-\sum_{a=1}^{N} \bar{\psi}_{f}^{a} \psi_{f}^{a}\right]},
$$

one can easily integrate out $\widetilde{A}$,

$$
\begin{aligned}
& Z^{\beta=2}=2^{N / 2}\left(\frac{\pi}{N}\right)^{N^{2} / 2} \frac{N}{\sqrt{2 \pi \alpha_{2}}} \int_{-\infty}^{\infty} \mathrm{d} x \exp \left[-\frac{N^{2}}{2} \frac{1-\alpha_{2}}{\alpha_{2}} x^{2}+2 i N k x\right] \\
& \times \frac{\int \mathrm{d} \psi \mathrm{d} \bar{\psi} \exp \left[-\sum_{a=1}^{N} \sum_{f, g=1}^{2 N_{\mathrm{f}}} \bar{\psi}_{f}^{a}\left(M-i x \mathbb{1}_{2 N_{\mathrm{f}}}\right)_{f g} \psi_{g}^{a}+\sum_{f, g=1}^{2 N_{\mathrm{f}}}\left(\sum_{a=1}^{N} \bar{\psi}_{f}^{a} \psi_{g}^{a}\right)\left(\sum_{b=1}^{N} \bar{\psi}_{g}^{b} \psi_{f}^{b}\right) /(2 N)\right]}{\int \mathrm{d} \psi_{f} \mathrm{~d} \bar{\psi}_{f} \exp \left[-\sum_{a=1}^{N} \sum_{f=1}^{2 N_{\mathrm{f}}} \bar{\psi}_{f}^{a} \psi_{f}^{a}\right]} .
\end{aligned}
$$

\footnotetext{
${ }^{9}$ The relation between the number of flavors $\tilde{N}_{\mathrm{f}}$ in [48] and our choice $N_{\mathrm{f}}$ is $\tilde{N}_{\mathrm{f}}=2 N_{\mathrm{f}}$.

${ }^{10}$ Matrix models having a similar structure were studied in [70-73].
} 
The quartic term in the fermions can be brought into bilinear form by means of the Hubbard-Stratonovich transformation. For this purpose we introduce an auxiliary Hermitian $2 N_{\mathrm{f}} \times 2 N_{\mathrm{f}}$ matrices $H$. This allows to integrate out the Grassmann variables leading to the result

$$
\begin{aligned}
& Z^{\beta=2}=2^{\left(N-2 N_{\mathrm{f}}\right) / 2}\left(\frac{\pi}{N}\right)^{\left(N^{2}-4 N_{\mathrm{f}}^{2}\right) / 2} \frac{N}{\sqrt{2 \pi \alpha_{2}}} \\
& \times \int_{-\infty}^{\infty} \mathrm{d} x \exp \left[-\frac{N^{2}}{2} \frac{1-\alpha_{2}}{\alpha_{2}} x^{2}+2 i N k x\right] \int \mathrm{d} H \exp \left[-\frac{N}{2} \operatorname{Tr} H^{2}\right] \operatorname{det}^{N}\left(H-i x \mathbb{1}_{2 N_{\mathrm{f}}}+M\right) .
\end{aligned}
$$

After shifting $H \rightarrow H+i x \mathbb{1}_{2 N_{\mathrm{f}}}$ via analytic deformation of the contours, we perform the $x$-integral for which we need the stricter bound $\alpha_{2}<N /\left(N+2 N_{\mathrm{f}}\right)$. Then, we obtain

$$
\begin{aligned}
Z^{\beta=2}= & 2^{\left(N-2 N_{\mathrm{f}}\right) / 2}\left(\frac{\pi}{N}\right)^{\left(N^{2}-4 N_{\mathrm{f}}^{2}\right) / 2} \sqrt{\frac{\widetilde{\alpha}_{2}}{\alpha_{2}}} \\
& \times \int \mathrm{d} H \exp \left[-\frac{N}{2} \widetilde{\alpha}_{2}(\operatorname{Tr} H-2 k)^{2}-\frac{N}{2} \operatorname{Tr} H^{2}\right] \operatorname{det}^{N}(H+M)
\end{aligned}
$$

with

$$
\frac{1}{\widetilde{\alpha}_{2}}=N \frac{1-\alpha_{2}}{\alpha_{2}}-2 N_{\mathrm{f}}>0 .
$$

This is the finite $N$ result that is still exact without any approximations. When employing the choice $(2.5)$ the parameter $\widetilde{\alpha}_{2}$ simplifies, i.e., $\widetilde{\alpha}_{2}=1$. In the following we keep $\widetilde{\alpha}_{2}$ fixed.

Let us consider the large- $N$ limit with $\widehat{M}=N M$ fixed. The integral is dominated by saddle point manifolds and fluctuations around them. When diagonalizing $H=U^{\dagger} \Lambda U$ with a real diagonal matrix $\Lambda=\operatorname{diag}\left(\lambda_{1}, \ldots, \lambda_{N_{\mathrm{f}}}\right)$, the saddle-point equation in the chiral limit $M=0$ reads

$$
\begin{array}{r}
0 \stackrel{!}{=} \frac{\partial}{\partial \lambda_{n}} S_{\Lambda} \quad \text { with } \quad S_{\Lambda} \equiv \frac{\widetilde{\alpha}_{2}}{2}\left(\sum_{i=1}^{2 N_{\mathrm{f}}} \lambda_{i}-2 k\right)^{2}+\frac{1}{2} \sum_{i=1}^{2 N_{\mathrm{f}}}\left(\lambda_{i}^{2}-\log \lambda_{i}^{2}\right) \\
\Longleftrightarrow \frac{1}{\lambda_{n}}-\lambda_{n}=\widetilde{\alpha}_{2}\left(\sum_{i=1}^{2 N_{\mathrm{f}}} \lambda_{i}-2 k\right) \quad \text { for } n=1, \cdots, 2 N_{\mathrm{f}}
\end{array}
$$

In general, there are multiple real solutions to this equation. However, we look for the minimum of the real part of $S_{\Lambda}$ which is achieved for $\Lambda=\Lambda_{k}$, cf. eq. (2.9), when $k \in \mathbb{Z}$ with $|k| \leq N_{\mathrm{f}}$. Indeed, the lower bound to the real part is

$$
\operatorname{Re} S_{\Lambda}=\frac{\widetilde{\alpha}_{2}}{2}\left(\sum_{i=1}^{2 N_{\mathrm{f}}} \lambda_{i}-2 k\right)^{2}+\sum_{i=1}^{2 N_{\mathrm{f}}}\left(\frac{1}{2} \lambda_{i}^{2}-\log \left|\lambda_{i}\right|\right) \stackrel{\widetilde{\alpha}_{2}>0}{\geq} \sum_{i=1}^{2 N_{\mathrm{f}}}\left(\frac{1}{2} \lambda_{i}^{2}-\log \left|\lambda_{i}\right|\right) \geq N_{\mathrm{f}}
$$

which is saturated only by $\Lambda_{k}$ and permutation of its diagonal elements. The second inequality follows from the fact that $\lambda_{i}^{2} / 2-\log \left|\lambda_{i}\right|$ is a concave function with its two minimums at $\lambda_{i}= \pm 1$. The fluctuations about $\Lambda_{k}$ give an overall constant, that comprises the sign $(-1)^{N k}$ from $\operatorname{det}^{N} \Lambda_{k}$, and can be incorporated exactly. As a result we obtain to first order in $M$,

$$
Z^{\beta=2} \sim \int_{\mathrm{U}\left(2 N_{\mathrm{f}}\right)} \mathrm{d} \mu(U) \operatorname{det}^{N}\left(\mathbb{1}_{N}+\frac{U^{\dagger} \Lambda_{k}^{-1} U \widehat{M}}{N}\right) \sim \int_{\mathrm{U}\left(2 N_{\mathrm{f}}\right)} \mathrm{d} \mu(U) \exp \left[\operatorname{Tr}\left(U \Lambda_{k} U^{\dagger} \widehat{M}\right)\right] .
$$


where we exploited that $\Lambda_{k}^{-1}=\Lambda_{k}$ because $k$ is an integer, cf. (2.9). This result holds regardless of the value of $\alpha_{2}$ as long as $\widetilde{\alpha}_{2}$ is fixed. The latter implies that $1-\alpha_{2}$ is of the order $1 / N$. The proper normalization of this partition function is computed in appendix A.2.

The result (2.28) realizes the symmetry breaking pattern (2.10) for the $\beta=2$ class. The integration for $U$ is effectively over the coset $\mathrm{U}\left(2 N_{\mathrm{f}}\right) /\left[\mathrm{U}\left(N_{\mathrm{f}}+k\right) \times \mathrm{U}\left(N_{\mathrm{f}}-k\right)\right]$. This modified symmetry breaking pattern is evidently enforced by the squared trace term in (2.24). The idea of using squared trace terms to constrain the symmetry realization is similar to the squared trace deformation in Polyakov-loop models [74-76]. The symmetry breaking patterns for $\beta=1$ and 4 in (2.10) are realized by the same mechanism.

Spectral sum rules for the eigenvalues of the Dirac operator can be derived by matching the quark mass expansion of the effective finite-volume partition function (2.28) with that of the partition function in $\mathrm{QCD}_{3}$. They have already been obtained in eq. (5.17) of [77] for general $k$ from a mathematical perspective. Adapting [77] to our convention, we obtain

$$
\left\langle\sum_{n} \frac{1}{\zeta_{n}}\right\rangle_{k}=\frac{i k}{N_{\mathrm{f}}}, \quad\left\langle\sum_{n} \frac{1}{\zeta_{n}^{2}}\right\rangle_{k}=\frac{2\left(N_{\mathrm{f}}^{2}-k^{2}\right)}{N_{\mathrm{f}}\left(4 N_{\mathrm{f}}^{2}-1\right)}, \quad\left\langle\left(\sum_{n} \frac{1}{\zeta_{n}}\right)^{2}\right\rangle_{k}=\frac{1-4 k^{2}}{4 N_{\mathrm{f}}^{2}-1}
$$

for a few low order sum-rules. Here $\left\{i \zeta_{n}\right\}$ are the Dirac eigenvalues rescaled by the average level spacing with $\zeta_{n} \in \mathbb{R}$, while $\langle\cdots\rangle_{k}$ denotes the average with respect to the $\mathrm{QCD}_{3}$ action with a Chern-Simons term at level $k$. These sum-rules are a direct consequence of the pattern of spontaneous symmetry breaking and are independent of the specific details of the random matrix model.

\subsection{Phase transitions at non-integer $k$}

As the symmetry breaking pattern changes when $k$ is shifted with unit increment, there must be a phase transition at non-integer values of $k$ for $|k|<N_{\mathrm{f}} .{ }^{11}$ To determine the locus of phase transitions, we have to solve the $2 N_{\mathrm{f}}$ coupled saddle point equations (2.26).

In the first step we consider

$$
\lambda_{0} \equiv-\frac{\widetilde{\alpha}_{2}}{2}\left(\sum_{n=1}^{2 N_{\mathrm{f}}} \lambda_{n}-2 k\right)
$$

as the $\left(2 N_{\mathrm{f}}+1\right)$ 'st variable. Then, the equations (2.26) for $\lambda_{1}, \ldots, \lambda_{2 N_{\mathrm{f}}}$ decouple and can be solved in terms of the auxiliary variable $\lambda_{0}$, yielding

$$
\lambda_{n}=\lambda_{0}+L_{n} \sqrt{\lambda_{0}^{2}+1}
$$

with $L_{n}= \pm 1$ a sign which is not fixed yet. The sum of these signs is denoted by $2 k_{L}=$ $\sum_{j=1}^{2 N_{\mathrm{f}}} L_{j}$, which plays the role of $2 k$ for a non-integer $k$. To obtain the solution for $\lambda_{0}$, we sum over all $n$ in (2.31) and find

$$
\sum_{n=1}^{2 N_{\mathrm{f}}} \lambda_{n}=2 k-\frac{2 \lambda_{0}}{\tilde{\alpha}_{2}}=2 N_{\mathrm{f}} \lambda_{0}+2 k_{L} \sqrt{\lambda_{0}^{2}+1}
$$

\footnotetext{
${ }^{11}$ Note that $k$ in the matrix model can be varied continuously even though $k$ in $\mathrm{QCD}_{3}$ is quantized to integers.
} 


\begin{tabular}{|c|cccccc|}
\hline$N_{\mathrm{f}}$ & {$[0,1]$} & {$[1,2]$} & {$[2,3]$} & {$[3,4]$} & {$[4,5]$} & {$[5,6]$} \\
\hline 0 & 0.52770 & - & - & - & - & - \\
1 & 0.50551 & 1.51674 & - & - & - & - \\
2 & 0.50237 & 1.50713 & 2.51194 & - & - & - \\
3 & 0.50132 & 1.50396 & 2.50661 & 3.50927 & - & - \\
4 & 0.50084 & 1.50252 & 2.50420 & 3.50589 & 4.50759 & - \\
5 & 0.50058 & 1.50175 & 2.50291 & 3.50408 & 4.50525 & 5.50642 \\
\hline
\end{tabular}

Table 1. Location of first-order phase transitions in each interval of $k$ for the Dyson index $\beta=2$ and the choice $\widetilde{\alpha}_{2}=1$, in particular we have chosen eq. (2.5) for the "Chern-Simons" coupling $\alpha_{2}$. The critical point in the interval $\left[N_{\mathrm{f}}, N_{\mathrm{f}}+1\right]$ is obtained by solving $(2.35)$ for $N_{\mathrm{f}}+\varepsilon$ with $\varepsilon \rightarrow 0$.

This equation has a unique real solution, because the right hand side plus $2 \lambda_{0} / \tilde{\alpha}_{2}$ is strictly monotonically increasing. The unique solution is

$$
\lambda_{0}=\frac{k\left(N_{\mathrm{f}}+1 / \tilde{\alpha}_{2}\right)-k_{L} \sqrt{\left(N_{\mathrm{f}}+1 / \tilde{\alpha}_{2}\right)^{2}+k^{2}-k_{L}^{2}}}{\left(N_{\mathrm{f}}+1 / \tilde{\alpha}_{2}\right)^{2}-k_{L}^{2}} .
$$

Summarizing, equations (2.31) and (2.33) yield all $2^{2 N_{\mathrm{f}}}$ saddle points. The solutions only depend on the still free integer $k_{L}=-N_{\mathrm{f}}, \ldots, N_{\mathrm{f}}$. The solutions for a fixed $k_{L}$ are $\left(\begin{array}{c}2 N_{\mathrm{f}} \\ N_{\mathrm{f}}+k_{L}\end{array}\right)$ degenerate. The real part of the action $S_{\Lambda}$ for a fixed $k_{L}$ is

$$
\begin{aligned}
\operatorname{Re}\left[S_{\Lambda}^{\left(k_{L}\right)}\right] & =\frac{2}{\widetilde{\alpha}_{2}} \lambda_{0}^{2}+\sum_{i=1}^{2 N_{\mathrm{f}}}\left\{\frac{1}{2}\left(\lambda_{0}+L_{i} \sqrt{\lambda_{0}^{2}+1}\right)^{2}-\log \left|\lambda_{0}+L_{i} \sqrt{\lambda_{0}^{2}+1}\right|\right\} \\
& =2\left(N_{\mathrm{f}}+\frac{1}{\widetilde{\alpha}_{2}}\right) \lambda_{0}^{2}+2 k_{L} \lambda_{0} \sqrt{\lambda_{0}^{2}+1}-2 k_{L} \log \left|\lambda_{0}+\sqrt{\lambda_{0}^{2}+1}\right|+N_{\mathrm{f}}
\end{aligned}
$$

This quantity has to be minimized in the integer $k_{L}=-N_{\mathrm{f}}, \ldots, N_{\mathrm{f}}$.

When $k$ is an integer with $|k| \leq N_{\mathrm{f}},(2.34)$ has a unique minimum at $k_{L}=k$ when $\lambda_{0}=0$. Note that this minimum is completely independent of $N_{\mathrm{f}}$ and $\widetilde{\alpha}_{2}$. Thus the discussion is valid for any number of flavors. The $N_{\mathrm{f}}$ and $\widetilde{\alpha}_{2}$ dependence enters the game when we studying the phase transition point with a real-valued $k$. Then we have to compare the actions $S_{\Lambda}^{(\lfloor k\rfloor)}$ and $S_{\Lambda}^{(\lceil k\rceil)}$ with the floor function $\lfloor$.$\rfloor and the ceiling function \lceil$.$\rceil yielding$ the largest integer smaller than or equal to $k$ and the smallest integer larger than or equal to $k$, respectively. The phase transition then happens when

$$
\operatorname{Re}\left[S_{\Lambda}^{(\lfloor k\rfloor)}\right]=\operatorname{Re}\left[S_{\Lambda}^{(\lceil k\rceil)}\right] .
$$

This is a transcendental equation in $k$ which always has one solution in each interval $[j, j+1]$ with $j=-N_{\mathrm{f}},-N_{\mathrm{f}}+1, \ldots, N_{\mathrm{f}}-1$. The change of $S_{\Lambda}^{(\lfloor k\rfloor)}$ to $S_{\Lambda}^{(\lceil k\rceil)}$ obviously exhibits a kink in the parameter $k_{L}$. Therefore these phase transitions are of first order. 
Phase transition points for general $N_{\mathrm{f}}$ are located symmetrically on the positive and negative sides of the $k$ axis, and hence it is sufficient to look for solutions to (2.35) for $k>0$. Table 1 is a summary for $1 \leq N_{\mathrm{f}} \leq 5$ with the Dyson index $\beta=2$ and the "ChernSimons" coupling $\alpha_{2}$ chosen as in (2.5). Only in the limit $N_{\mathrm{f}} \rightarrow \infty$ do the half integers $k=n+\frac{1}{2}(n \in \mathbb{Z})$ become the phase transition points. For a finite number of flavors we get corrections, which can be computed via a large- $N_{\mathrm{f}}$ expansion of $(2.35)$ and the solution $k=(n+1 / 2) \sum_{j=0}^{\infty} c_{j} /\left(N_{\mathrm{f}}+1 / \tilde{\alpha}_{2}\right)^{j}$. Assuming $n=\mathcal{O}(1)$, the corrected transition points are

$$
k=\left[1+\frac{1}{24} \frac{1}{\left(N_{\mathrm{f}}+1 / \tilde{\alpha}_{2}\right)^{2}}+\frac{17}{1920} \frac{1}{\left(N_{\mathrm{f}}+1 / \tilde{\alpha}_{2}\right)^{4}}+\mathcal{O}\left(\left(N_{\mathrm{f}}+1 / \tilde{\alpha}_{2}\right)^{-6}\right)\right]\left(n+\frac{1}{2}\right)
$$

for $n \in \mathbb{Z}$. The residue $\mathcal{O}\left(\left(N_{\mathrm{f}}+1 / \tilde{\alpha}_{2}\right)^{-6}\right)$ may depend on $n$, as well, though it seems to be a very weak dependence.

\subsection{Partition function at finite and large $N$}

In this section, we evaluate the partition function at finite $N$ and use this result to derive its large- $N$ limit. This discussion serves two purposes. First and foremost, the finite $N$ results enable us to study the approach to the thermodynamic limit. Second, it provides an independent consistency check of the large $N$ result (2.28).

The partition function (2.19) is a one-parameter integral over a GUE partition function with $2 N_{\mathrm{f}}$ flavors. The latter will be rewritten using the identity [78, 79],

$$
\begin{gathered}
\frac{\int \mathrm{d} A \exp \left[-\frac{N}{2} \operatorname{Tr} A^{2}\right] \prod_{\ell=1}^{L}\left[\operatorname{det}\left(\lambda_{\ell} \mathbb{1}_{N}-A\right) \operatorname{det}\left(\mu_{\ell} \mathbb{1}_{N}-A\right)\right]}{\int \mathrm{d} A \exp \left[-\frac{N}{2} \operatorname{Tr} A^{2}\right]} \\
=\frac{C_{N, L}}{\Delta_{L}(\lambda) \Delta_{L}(\mu)} \operatorname{det}_{1 \leq a, b \leq L}\left[\mathcal{K}_{N+L}^{(N)}\left(\lambda_{a}, \mu_{b}\right)\right]
\end{gathered}
$$

where the integration is over hermitian $N \times N$ matrices, and we employ the notation $\Delta_{L}($. for the Vandermonde determinant with the exceptional case $\Delta_{1} \equiv 1$. The kernel is given by

$$
\mathcal{K}_{j}^{(N)}\left(\lambda_{a}, \mu_{b}\right) \equiv \frac{P_{j}^{(N)}\left(\lambda_{a}\right) P_{j-1}^{(N)}\left(\mu_{b}\right)-P_{j}^{(N)}\left(\mu_{b}\right) P_{j-1}^{(N)}\left(\lambda_{a}\right)}{\lambda_{a}-\mu_{b}}
$$

with the monic polynomials

$$
P_{\ell}^{(N)}(s) \equiv \frac{1}{(2 N)^{\ell / 2}} H_{\ell}\left(\sqrt{\frac{N}{2}} s\right), \quad \ell=0,1,2, \ldots
$$

where $H_{\ell}(x)=\mathrm{e}^{x^{2}}(-\partial)^{\ell} \mathrm{e}^{-x^{2}}$ are the Hermite polynomials. The polynomials $P_{\ell}^{(N)}(s)$ satisfy the orthogonality relation

$$
\int_{-\infty}^{\infty} \mathrm{d} s \mathrm{e}^{-N s^{2} / 2} P_{\ell}^{(N)}(s) P_{m}^{(N)}(s)=\delta_{\ell m} h_{m}^{(N)}, \quad h_{m}^{(N)} \equiv \frac{\sqrt{2 \pi} m !}{N^{m+\frac{1}{2}}},
$$

and the normalization constant is given by

$$
C_{N, L} \equiv\left[h_{N+L-1}^{(N)}\right]^{-L} \prod_{i=N}^{N+L-1} h_{i}^{(N)}
$$


In our case we have $2 N_{\mathrm{f}}$ flavors, each with its own mass $m_{f}$, so that we have no natural splitting into two sets of masses. We choose $\lambda_{j}=x+i m_{j}$ and $\mu_{j}=x+i \widetilde{m}_{j} \equiv x+i m_{N_{\mathrm{f}}+j}$ for $j=1, \cdots, N_{\mathrm{f}}$. Applying (2.37) to the partition function (2.19), we obtain, up to an irrelevant normalization,

$$
\begin{gathered}
Z^{\beta=2} \sim(-1)^{N_{\mathrm{f}} N} \int_{-\infty}^{\infty} \mathrm{d} x \frac{\exp \left[-N\left(N_{\mathrm{f}}+\frac{1}{2}\right) x^{2}+2 i N k x\right]}{\Delta_{N_{\mathrm{f}}}(x+i m) \Delta_{N_{\mathrm{f}}}(x+i \widetilde{m})} \operatorname{det}_{1 \leq a, b \leq N_{\mathrm{f}}}\left[\mathcal{K}_{N+N_{\mathrm{f}}}^{(N)}\left(x+i m_{a}, x+i \widetilde{m}_{b}\right)\right] \\
\sim \frac{(-1)^{N_{\mathrm{f}}\left(N_{\mathrm{f}}-1\right) / 2+N_{\mathrm{f}} N}}{\Delta_{N_{\mathrm{f}}}(m) \Delta_{N_{\mathrm{f}}}(\widetilde{m})} \int_{-\infty}^{\infty} \mathrm{d} x \exp \left[-N\left(N_{\mathrm{f}}+\frac{1}{2}\right) x^{2}+2 i N k x\right] \\
\quad \times \operatorname{det}_{1 \leq a, b \leq N_{\mathrm{f}}}\left[\frac{H_{N+N_{\mathrm{f}}}\left(\sqrt{\frac{N}{2}}\left(x+i m_{a}\right)\right) H_{N+N_{\mathrm{f}}-1}\left(\sqrt{\frac{N}{2}}\left(x+i \widetilde{m}_{b}\right)\right)}{i\left(m_{a}-\widetilde{m}_{b}\right)}\right],
\end{gathered}
$$

where we used the translation symmetry and homogeneity of the Vandermonde determinant $\Delta_{N_{\mathrm{f}}}(x+i m)=\Delta_{N_{\mathrm{f}}}(i m)=i^{N_{\mathrm{f}}\left(N_{\mathrm{f}}-1\right) / 2} \Delta_{N_{\mathrm{f}}}(m)$ and likewise for $\widetilde{m}$. The sign $(-1)^{N_{\mathrm{f}} N}$ results from pulling a factor $i$ out of each determinant in (2.19).

In the simplest case $k=0$, due to the Gaussian factor, the variable $x$ scales as $\mathcal{O}(1 / \sqrt{N})$ while the masses $m$ and $\widetilde{m}$ are of order $\mathcal{O}(1 / N)$. When exploiting the asymptotic form,

$$
H_{N}(t) \sim \mathrm{e}^{t^{2} / 2} \cos \left[\left(2 N+\frac{1}{2}\right) \frac{t}{\sqrt{2 N}}-\frac{N \pi}{2}\right]
$$

at $N \gg 1$ and $t=\mathcal{O}(1)$, we obtain

$$
\begin{aligned}
& \frac{H_{N+N_{\mathrm{f}}}\left(\sqrt{\frac{N}{2}}\left(x+i m_{a}\right)\right) H_{N+N_{\mathrm{f}}-1}\left(\sqrt{\frac{N}{2}}\left(x+i \widetilde{m}_{b}\right)\right)}{i\left(m_{a}-\widetilde{m}_{b}\right)} \\
& -\frac{H_{N+N_{\mathrm{f}}}\left(\sqrt{\frac{N}{2}}\left(x+i \widetilde{m}_{b}\right)\right) H_{N+N_{\mathrm{f}}-1}\left(\sqrt{\frac{N}{2}}\left(x+i m_{a}\right)\right)}{i\left(m_{a}-\widetilde{m}_{b}\right)} \sim \mathrm{e}^{N x^{2} / 2} \frac{\sinh \left[N\left(m_{a}-\widetilde{m}_{b}\right)\right]}{m_{a}-\widetilde{m}_{b}} .
\end{aligned}
$$

Thereupon, the integral over $x$ factorizes, and we are left with the simpler expression,

$$
Z^{\beta=2} \sim \frac{(-1)^{N_{\mathrm{f}}\left(N_{\mathrm{f}}-1\right) / 2+N_{\mathrm{f}} N}}{\Delta_{N_{\mathrm{f}}}(m) \Delta_{N_{\mathrm{f}}}(\widetilde{m})} \operatorname{det}_{1 \leq a, b \leq N_{\mathrm{f}}}\left\{\frac{\sinh \left[N\left(m_{a}-\widetilde{m}_{b}\right)\right]}{m_{a}-\widetilde{m}_{b}}\right\}
$$

For a parity-invariant mass $\widetilde{m}=-m$ it coincides with the partition function obtained previously $[45,46]$.

One can also obtain the result (2.45) from the low-energy limit of the partition function given in (2.6) for $k=0$. For this purpose we exploit the parameterization [80, 81]

$$
\begin{aligned}
& U^{\dagger} \Lambda_{0} U=\operatorname{diag}\left(U_{1}^{\dagger}, U_{2}^{\dagger}\right)\left(\begin{array}{c}
\cos \Phi \sin \Phi \\
\sin \Phi \cos \Phi
\end{array}\right) \operatorname{diag}\left(U_{1}, U_{2}\right), \\
& \text { with } \Phi=\operatorname{diag}\left(\varphi_{1}, \ldots, \varphi_{N_{\mathrm{f}}}\right) \in[0, \pi]^{N_{\mathrm{f}}} \text { and } U_{1}, U_{2} \in \mathrm{U}\left(N_{\mathrm{f}}\right) .
\end{aligned}
$$



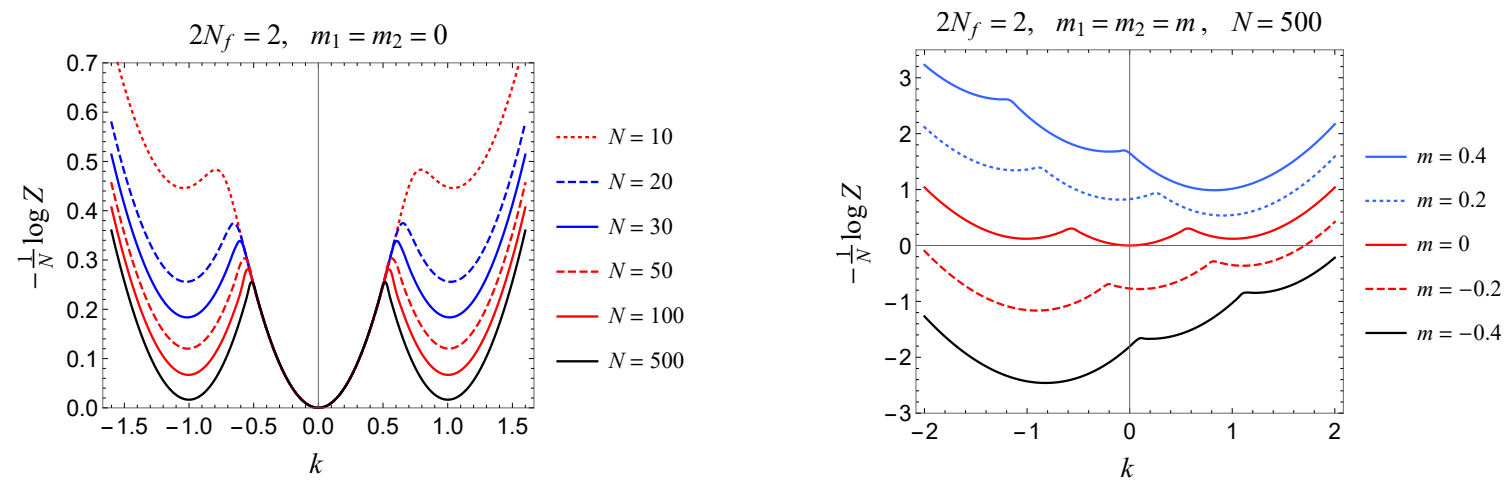

Figure 2. The free energy for two flavors with equal mass in the chiral limit for varying $N$ for zero mass (left) and various masses for $N=100$ (right). The value at $k=0$ is subtracted for each $N$. The spacing between curves in the right plot has been adjusted by hand for better visibility.

The measure is then

$$
\mathrm{d} \mu(U) \sim \Delta_{N_{\mathrm{f}}}^{2}(\cos \Phi) \prod_{j=1}^{N_{\mathrm{f}}} \sin \varphi_{j} \mathrm{~d} \varphi_{j} \mathrm{~d} \mu\left(U_{1}\right) \mathrm{d} \mu\left(U_{2}\right),
$$

and the Lagrangian takes the form

$$
\operatorname{Tr} U^{\dagger} \Lambda_{0} U \widehat{M}=N\left(\operatorname{Tr} U_{1}^{\dagger} \cos \Phi U_{1} m-\operatorname{Tr} U_{2}^{\dagger} \cos \Phi U_{2} \widetilde{m}\right) .
$$

The integrals over $U_{1}$ and $U_{2}$ are each Harish-Chandra-Itzykson-Zuber integrals [82, 83],

$$
\int \mathrm{d} \mu\left(U_{1}\right) \exp \left[N \operatorname{Tr} U_{1}^{\dagger} \cos \Phi U_{1} m\right] \sim \frac{\operatorname{det}_{1 \leq a, b \leq N_{\mathrm{f}}}\left[\exp \left[N m_{a} \cos \varphi_{b}\right]\right]}{\Delta_{N_{\mathrm{f}}}(m) \Delta_{N_{\mathrm{f}}}(\cos \Phi)}
$$

and similar for $\widetilde{m}$. The remaining integrals in

$$
Z^{\beta=2} \sim \int \prod_{j=1}^{N_{\mathrm{f}}} \sin \varphi_{j} \mathrm{~d} \varphi_{j} \frac{\operatorname{det}_{1 \leq a, b \leq N_{\mathrm{f}}}\left[\exp \left[N m_{a} \cos \varphi_{b}\right]\right] \operatorname{det}_{1 \leq a, b \leq N_{\mathrm{f}}}\left[\exp \left[-N \widetilde{m}_{a} \cos \varphi_{b}\right]\right]}{\Delta_{N_{\mathrm{f}}}(m) \Delta_{N_{\mathrm{f}}}(\widetilde{m})}
$$

over $\Phi$ can be performed with the Andréief identity [84] yielding (2.45).

Let us examine the simplest case of two flavors $\left(2 N_{\mathrm{f}}=2\right)$ with a $\mathrm{U}(2)$-invariant mass $m_{1}=\widetilde{m}_{1}=m$. Then, eq. (2.42) simplifies to

$$
\begin{aligned}
Z_{2 N_{\mathrm{f}}=2}^{\beta=2}(m ; k) \sim & (-1)^{N_{\mathrm{f}} N+1} \mathrm{e}^{N m(4 k+3 m) / 2} \\
& \times \int_{-\infty}^{\infty} \mathrm{d} x \mathrm{e}^{-3 x^{2}} \cos (\sqrt{2 N}(2 k+3 m) x)\left[H_{N+2}(x) H_{N}(x)-H_{N+1}(x)^{2}\right]
\end{aligned}
$$

after substituting $x \rightarrow \sqrt{2 / N} x-i m$. This integral can be carried out with the help of a formula in [85, section 7.374 , eq. 9$]$, with the result

$$
Z_{2 N_{\mathrm{f}}=2}^{\beta=2}(m ; k) \sim \mathrm{e}^{-2 N k^{2} / 3} \sum_{\ell=1}^{N+1} \frac{3^{\ell}}{(\ell-1) !(N+2-\ell) !(N+1-\ell) !} H_{2 N+2-2 \ell}\left(\frac{i \sqrt{3 N}(2 k+3 m)}{6}\right) .
$$



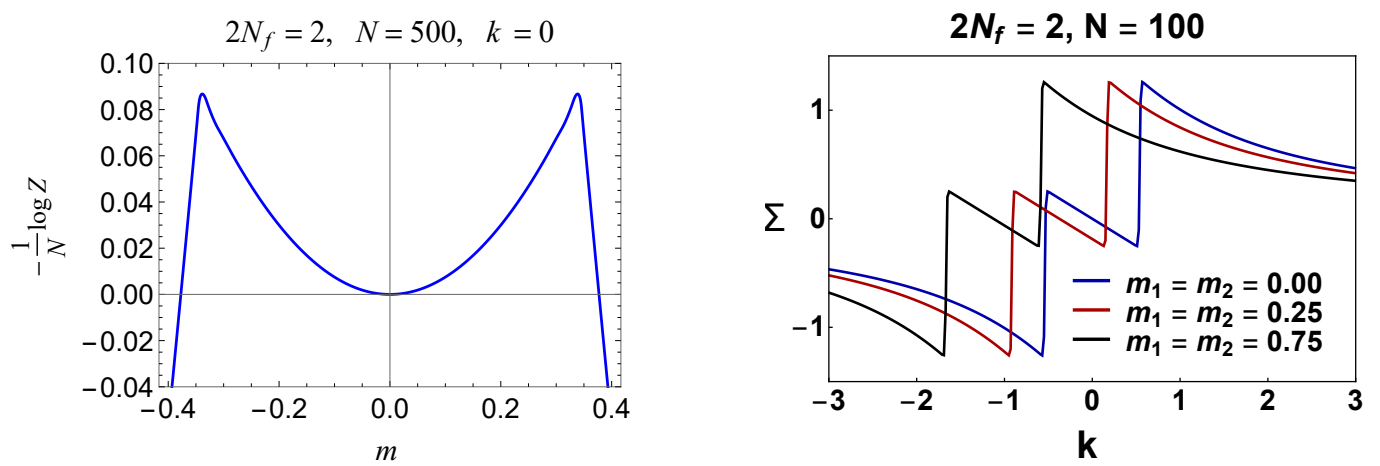

Figure 3. The mass dependence of the free energy for $k=0$ for two flavors with mass $\left(m_{1}, m_{2}\right)=$ $(m, m)$ (left) and the $k$ dependence of the chiral condensate, $\Sigma$, for three different values of the mass also for two flavors with equal mass.

This form is suited for fast numerical evaluation. Since $Z(m ; k)=Z(-m ;-k)$, one may assume $m \geq 0$ without loss of generality. The $k$ dependence of the partition function can now be investigated via (2.52).

Figure 2 (left) displays the evolution of the free energy with $N$ in the microscopic limit. Evidently there are quite strong deviations from the large $N$ limit even for rather moderate matrix sizes like $N=50$ which usually yields close to perfect agreement for the microscopic level density. As $N$ grows, kinks appear that get sharper. They represent first order transitions in the thermodynamical limit. The region around the origin is the flavor symmetry broken phase $\mathrm{U}(2) \rightarrow \mathrm{U}(1) \times \mathrm{U}(1)$ with massless Nambu-Goldstone modes. The other two regions are the symmetry-restored phases. The right plot of figure 2 illustrates the shift of the phase transition points as a function of the masses that are of order $\mathcal{O}(1)$ instead of $\mathcal{O}(1 / N)$, and hence they are outside the microscopic domain. The two kinks move towards negative $k$ (positive $k)$ for $m>0(m<0)$, respectively.

Figure 3 (left plot) shows the mass dependence of the free energy at $k=0$, again at an $N$ independent mass, i.e., $N m \gg 1$. The two pronounced kinks at $m \approx \pm 0.34$ indicate a strong first-order transition that corresponds to the passage of a kink over the origin in the right plot of figure 2. The middle region corresponds to a symmetry-broken phase with massless Nambu-Goldstone bosons ${ }^{12}$ whereas the outer regions are symmetric gapped phases. Such phase transitions at nonzero masses were argued to exist in [48] and our matrix model serves as a toy model for this phenomenon.

Finally, in figure 3 (right) we show the $k$ dependence of the quark-antiquark condensate defined as

$$
\Sigma=\frac{1}{2 N} \frac{\partial}{\partial m} \log Z(m ; k)
$$

for three different values of the mass, $m_{1}=m_{2}=0, m_{1}=m_{2}=0.25$ and $m_{1}=m_{2}=0.75$. Hence, also in figure 3 we do not show the microscopic limit of the chiral condensate. The

\footnotetext{
${ }^{12}$ Note that the pions remain massless in the presence of flavor-symmetric fermion masses. This is an important difference from four-dimensional QCD where the quark mass inevitably breaks the flavor symmetry of quarks.
} 
masses are of order $\mathcal{O}(1)$ and not $\mathcal{O}(1 / N)$. In the microscopic limit the two kinks are at exactly the same position as in figure 2 (left). The condensate has still a discontinuity at the values of $k$ for which the free energy has a kink for masses which are of order one. Yet, for increasing mass the kinks move to infinity, approximately as $\sim \frac{3}{2} m$, and the quenched result is recovered for $m \rightarrow \infty$. For masses with opposite sign, $m_{1}=-m_{2}$, the $k$-dependence of the free energy remains symmetric about zero, but the kinks move away from zero, again like $\sim \frac{3}{2} m$ for large $m$, so that for $m \rightarrow \infty$ the quenched limit is recovered.

\section{Spectral correlation functions}

This section is mostly devoted to the level density, the quark-antiquark condensate and their microscopic large- $N$ limit, though in subsection 3.1 we also study all $k$-point correlation functions at finite $N$. In this subsection we obtain an exact expression for the spectral density at finite $N$. To illustrate what happens when $N$ is increasing at fixed Chern-Simons coupling $k$, we consider the quenched level density $\left(N_{\mathrm{f}}=0\right)$ at finite $N$ in subsection 3.2. This result is amenable to a saddle point approximation which allows us to obtain the microscopic limit of the spectral density (see section 3.3) defined as

$$
\widehat{R}(\widehat{\lambda} ; k) \equiv \lim _{N \rightarrow \infty} \frac{1}{N} R\left(\frac{\widehat{\lambda}}{N} ; k\right) .
$$

For more flavors the microscopic limit can be derived much more easily from an expression where the spectral density is given by a ratio of an $N_{\mathrm{f}}+2$ flavor partition function and an $N_{\mathrm{f}}$ flavor partition function as is discussed in section 2.5. Starting from this result we obtain in section 3.4 the microscopic spectral density for $N_{\mathrm{f}}$ flavors. In particular, we will work out the one-flavor case in detail.

For $N_{\mathrm{f}}=1$ the partition function is given by the sum of three saddle points (see section 2.4)

$$
Z_{N_{\mathrm{f}}=1}\left(m_{1}, m_{2} ; k\right)=Z_{N_{\mathrm{f}}=1, k_{L}=-1}\left(m_{1}, m_{2} ; k\right)+Z_{N_{\mathrm{f}}=1, k_{L}=0}\left(m_{1}, m_{2} ; k\right)+Z_{N_{\mathrm{f}}=1, k_{L}=1}\left(m_{1}, m_{2} ; k\right) .
$$

Because of the reweighted structure of the partition function, the spectral density is given by

$$
R(\lambda ; k)=\frac{1}{Z_{N_{\mathrm{f}}=1}\left(m_{1}, m_{2} ; k\right)} \sum_{k_{L}=-N_{\mathrm{f}}}^{N_{\mathrm{f}}}\left[Z_{N_{\mathrm{f}}=1, k_{L}}\left(m_{1}, m_{2} ; k\right) R_{N_{\mathrm{f}}=1, k_{L}}(\lambda ; k)\right]
$$

with the level density corresponding to the saddle point $k_{L}$ given by

$$
R_{N_{\mathrm{f}}=1, k_{L}}(\lambda ; k)=\frac{\left\langle\sum_{k} \delta\left(\lambda-\lambda_{k}\right)\right\rangle_{Z_{N_{\mathrm{f}}=1, k_{L}}}}{Z_{N_{\mathrm{f}}=1, k_{L}}} .
$$

Since the partition functions behave as

$$
Z_{N_{\mathrm{f}}=1, k_{L}} \sim e^{-N f_{k_{L}}},
$$

where $f_{k_{L}}$ is the free energy of the $k_{L}$ 'th saddle point, we have that in the large- $N$ limit, the spectral density is dominated by one saddle point (unless $k$ is exactly at the phase transition point). To derive the results of subsection 3.4, we make use of results obtained in appendix A. 


\subsection{General $N_{\text {f }}$ at finite $N$}

The matrix model approach gives us a way to investigate spectral fluctuations of the Dirac operator in $\mathrm{QCD}_{3}$. The simplest way to compute the $n$-point correlation functions $R\left(\lambda_{1}, \ldots, \lambda_{n} ; M ; k\right)$ of the matrix $A$ in the ensemble (2.1) would be to make use of the reweighted structure $(2.19)$. If $\widetilde{R}\left(\lambda_{1}, \ldots, \lambda_{n} ; M ; x\right)$ is the $n$-point spectral correlation function of $A$ for the GUE ensemble $\mathcal{Z}_{N, N_{\mathrm{f}}}(M-i x)$ with $2 N_{\mathrm{f}}$ flavors, we simply have

$$
R\left(\lambda_{1}, \ldots, \lambda_{n} ; M ; k\right) \sim \frac{\int_{-\infty}^{\infty} \mathrm{d} x \mathrm{e}^{-N\left(N_{\mathrm{f}}+\frac{1}{2}\right) x^{2}+2 i N k x} \mathcal{Z}_{N, N_{\mathrm{f}}}(M-i x) \widetilde{R}\left(\lambda_{1}, \ldots, \lambda_{n} ; M ; x\right)}{\int_{-\infty}^{\infty} \mathrm{d} x \mathrm{e}^{-N\left(N_{\mathrm{f}}+\frac{1}{2}\right) x^{2}+2 i N k x} \mathcal{Z}_{N, N_{\mathrm{f}}}(M-i x)}
$$

The spectral correlation functions corresponding to the partition function $\mathcal{Z}_{N, N_{\mathrm{f}}}(M-i x)$ have been computed in [36, 37] for the massless case, and in [38, 39, 45, 46] for nonzero masses with pairwise opposite signs. By substituting the results from $[38,39,45,46]$ into $\widetilde{R}$ we obtain the spectral functions for our matrix model.

In this paper we only explicitly work out the one-point function (spectral density) for arbitrary $2 N_{\mathrm{f}}$ masses. Recalling the shift $A \rightarrow A-x \mathbb{1}_{N}$, we get from (2.20)

$$
\begin{aligned}
& \widetilde{R}(\lambda ; M ; x)=\frac{1}{\mathcal{Z}_{N, N_{\mathrm{f}}}(M-i x)} \int \mathrm{d} A \operatorname{Tr} \delta(\lambda+x-A) \mathrm{e}^{-\frac{N}{2} \operatorname{Tr} A^{2}} \prod_{f=1}^{2 N_{\mathrm{f}}} \operatorname{det}\left[i A+\left(m_{f}-i x\right) \mathbb{1}_{N}\right] \\
& \sim \frac{(-1)^{N_{\mathrm{f}} N}}{\mathcal{Z}_{N, N_{\mathrm{f}}}(M-i x)} \int_{\mathbb{R}^{N}} \mathrm{~d} a_{1} \cdots \mathrm{d} a_{N} \delta\left(\lambda+x-a_{N}\right) \mathrm{e}^{-\frac{N}{2} \sum_{n=1}^{N} a_{n}^{2}} \Delta_{N}^{2}(a) \prod_{f=1}^{2 N_{\mathrm{f}}} \prod_{n=1}^{N}\left(a_{n}-\left(x+i m_{f}\right)\right) \\
& =\frac{(-1)^{N_{\mathrm{f}} N} \mathrm{e}^{-\frac{N}{2}(\lambda+x)^{2}} \prod_{f=1}^{2 N_{\mathrm{f}}}\left(\lambda-i m_{f}\right)}{\mathcal{Z}_{N, N_{\mathrm{f}}}(M-i x)} \int_{\mathbb{R}^{N-1}} \mathrm{~d} a_{1} \cdots \mathrm{d} a_{N-1} \mathrm{e}^{-\frac{N}{2} \sum_{n=1}^{N-1} a_{n}^{2}} \Delta_{N-1}^{2}(a) \\
& \quad \times \prod_{n=1}^{N-1}\left[\left(a_{n}-x-\lambda\right)^{2} \prod_{f=1}^{2 N_{\mathrm{f}}}\left(a_{n}-\left(x+i m_{f}\right)\right)\right] .
\end{aligned}
$$

The remaining integral is nothing but the partition function of GUE with $2 N_{\mathrm{f}}+2$ flavors. Thus, it can be expressed as an integral over a hermitian $(N-1) \times(N-1)$ matrix $B$,

$$
\begin{aligned}
\widetilde{R}(\lambda ; M ; x) \sim & (-1)^{N_{\mathrm{f}}-N+1} \frac{\mathrm{e}^{-\frac{N}{2}(\lambda+x)^{2}} \prod_{f=1}^{2 N_{\mathrm{f}}}\left(\lambda-i m_{f}\right)}{\mathcal{Z}_{N, N_{\mathrm{f}}}(M-i x)} \\
& \times \int \mathrm{d} B \mathrm{e}^{-\frac{N}{2} \operatorname{Tr} B^{2}} \prod_{f=1}^{2 N_{\mathrm{f}}+2} \operatorname{det}\left[i B+\left(m_{f}-i x\right) \mathbb{1}_{N-1}\right]
\end{aligned}
$$

where we have defined $m_{2 N_{\mathrm{f}}+1}=m_{2 N_{\mathrm{f}}+2}=-i \lambda$. This matrix integral can be computed with the help of (2.37). In doing so, the degeneracy of $m_{2 N_{\mathrm{f}}+1}$ and $m_{2 N_{\mathrm{f}}+2}$ must be lifted slightly to avoid an apparent singularity in (2.37). Labeling

$$
\begin{aligned}
\left(\kappa_{1}, \cdots, \kappa_{N_{\mathrm{f}}}, \kappa_{N_{\mathrm{f}}+1}\right) & \equiv\left(m_{1}, \cdots, m_{N_{\mathrm{f}}},-i \lambda\right), \\
\left(\widetilde{\kappa}_{1}, \cdots, \widetilde{\kappa}_{N_{\mathrm{f}}}, \widetilde{\kappa}_{N_{\mathrm{f}}+1}\right) & \equiv\left(\widetilde{m}_{1}, \cdots, \widetilde{m}_{N_{\mathrm{f}}},-i \lambda+\varepsilon\right)=\left(m_{N_{\mathrm{f}}+1}, \cdots, m_{2 N_{\mathrm{f}}},-i \lambda+\varepsilon\right), \\
\varepsilon & =0^{+}
\end{aligned}
$$


and combining (2.37), (2.42), (3.6) and (3.8), the spectral density is finally obtained as

$$
\begin{aligned}
& R(\lambda ; M ; k) \sim \mathrm{e}^{-\frac{N}{2} \lambda^{2}} \prod_{f=1}^{2 N_{\mathrm{f}}}\left(\lambda-i m_{f}\right) \\
& \times \lim _{\varepsilon \rightarrow 0} \frac{\frac{(-1)^{\left(N_{\mathrm{f}}+1\right) N_{\mathrm{f}} / 2}}{\Delta \Delta_{\mathrm{f}}+1(\kappa) \Delta_{\mathrm{f}_{\mathrm{f}}+1}(\widetilde{\kappa})} \int_{-\infty}^{\infty} \mathrm{d} x \mathrm{e}^{-N\left[\left(N_{\mathrm{f}}+1\right) x^{2}-(2 i k-\lambda) x\right]} \operatorname{det}_{1 \leq a, b \leq N_{\mathrm{f}}+1}\left[\mathcal{K}_{N+N_{\mathrm{f}}}^{(N)}\left(x+i \kappa_{a}, x+i \widetilde{\kappa}_{b}\right)\right]}{\frac{(-1)^{N_{\mathrm{f}}\left(N_{\mathrm{f}}-1\right) / 2}}{\Delta_{N_{\mathrm{f}}}(m) \Delta_{N_{\mathrm{f}}}(\tilde{m})} \int_{-\infty}^{\infty} \mathrm{d} x \mathrm{e}^{-N\left[\left(N_{\mathrm{f}}+\frac{1}{2}\right) x^{2}-2 i k x\right]} \operatorname{det}_{1 \leq a, b \leq N_{\mathrm{f}}}\left[\mathcal{K}_{N+N_{\mathrm{f}}}^{(N)}\left(x+i m_{a}, x+i \widetilde{m}_{b}\right)\right]} \\
& \sim(-1)^{N_{\mathrm{f}}} \mathrm{e}^{-\frac{N}{2} \lambda^{2}} \\
& \times \frac{\int_{-\infty}^{\infty} \mathrm{d} x \mathrm{e}^{-N\left[\left(N_{\mathrm{f}}+1\right) x^{2}-(2 i k-\lambda) x\right]} \operatorname{det}\left[\begin{array}{cc}
\left\{\mathcal{K}_{N+N_{\mathrm{f}}}^{(N)}\left(x+i m_{a}, x+i \widetilde{m}_{b}\right)\right\}_{\substack{1 \leq a \leq N_{\mathrm{f}} \\
1 \leq b \leq N_{\mathrm{f}}}}\left\{\mathcal{K}_{N+N_{\mathrm{f}}}^{(N)}\left(x+i m_{a}, x+\lambda\right)\right\}_{1 \leq a \leq N_{\mathrm{f}}} \\
\left\{\mathcal{K}_{N+N_{\mathrm{f}}}^{(N)}\left(x+\lambda, x+i \widetilde{m}_{b}\right)\right\}_{1 \leq b \leq N_{\mathrm{f}}} & {[\partial \mathcal{K}]_{N+N_{\mathrm{f}}}^{(N)}(x+\lambda)}
\end{array}\right]}{\int_{-\infty}^{\infty} \mathrm{d} x \mathrm{e}^{-N\left[\left(N_{\mathrm{f}}+\frac{1}{2}\right) x^{2}-2 i k x\right]} \operatorname{det}_{1 \leq a, b \leq N_{\mathrm{f}}}\left[\mathcal{K}_{N+N_{\mathrm{f}}}^{(N)}\left(x+i m_{a}, x+i \widetilde{m}_{b}\right)\right]},
\end{aligned}
$$

where we employ the notation

$$
[\partial \mathcal{K}]_{n}^{(N)}(\mu) \equiv \lim _{\lambda \rightarrow \mu} \mathcal{K}_{n}^{(N)}(\lambda, \mu)=\frac{1}{2(2 N)^{n-1}}\left[H_{n}^{2}\left(\sqrt{\frac{N}{2}} \mu\right)-H_{n+1}\left(\sqrt{\frac{N}{2}} \mu\right) H_{n-1}\left(\sqrt{\frac{N}{2}} \mu\right)\right] .
$$

The overall normalization of $R$ is fixed by $\int_{-\infty}^{\infty} \lambda R(\lambda ; M ; k)=N$. Equation (3.10) implies that for real masses the complex conjugate of the level density acts as a reflection of the spectrum, i.e.,

$$
[R(\lambda ; M ; k)]^{*}=R(-\lambda ; M ; k) .
$$

Hence, $\operatorname{Re}[R(\lambda ; M ; k)]$ is an even function of $\lambda$ while $\operatorname{Im}[R(\lambda ; M ; k)]$ is odd.

Another representation of the level density, which is convenient for the derivation of the microscopic limit, is

$$
\begin{aligned}
R(\lambda ; M ; k)= & \frac{(-\pi)^{N-1}}{(N-1) !} \mathrm{e}^{-\frac{N}{2} \lambda^{2}} \prod_{f=1}^{2 N_{\mathrm{f}}}\left(i \lambda+m_{f}\right) \\
& \times \frac{\int \mathrm{d} B \exp \left[\frac{\alpha_{2}}{2}(\operatorname{Tr} B+\lambda-2 i k)^{2}-\frac{N}{2} \operatorname{Tr} B^{2}\right] \prod_{f=1}^{2 N_{\mathrm{f}}+2} \operatorname{det}\left[i B+m_{f} \mathbb{1}_{N-1}\right]}{\int \mathrm{d} A \exp \left[\frac{\alpha_{2}}{2}(\operatorname{Tr} A-2 i k)^{2}-\frac{N}{2} \operatorname{Tr} A^{2}\right] \prod_{f=1}^{2 N_{\mathrm{f}}} \operatorname{det}\left[i A+m_{f} \mathbb{1}_{N}\right]} .
\end{aligned}
$$

This result is obtained by shifting back to $A \rightarrow A+x \mathbb{1}_{N}$ and $B \rightarrow B+x \mathbb{1}_{N-1}$ and then integrating over $x$ in the denominator as well as in the numerator. The normalization follows from integration over $\lambda$ and combining this integral with the $B$-integral to the $A$ integral in the denominator. We recall the value of $\alpha_{2}=N /\left(N+2 N_{\mathrm{f}}+1\right)$, cf. (2.5). Indeed this result could be directly derived from the partition function (2.1), by setting one of the eigenvalues of $A$ equal to $\lambda$.

When additionally shifting $B \rightarrow B+\lambda^{\prime} \mathbb{1}_{N-1}$ with $\lambda^{\prime}=\lambda /\left(2 N_{\mathrm{f}}+2\right)$ in eq. (3.13), we reduce the level density to a quotient of two almost identical partition functions,

$$
\begin{aligned}
R(\lambda ; M ; k)= & (-1)^{N-1} \frac{\pi^{N-1}}{(N-1) !} \exp \left[-\frac{\left(2 N_{\mathrm{f}}+1\right) N}{4\left(N_{\mathrm{f}}+1\right)} \lambda^{2}-i \frac{N}{N_{\mathrm{f}}+1} k \lambda\right] \prod_{f=1}^{2 N_{\mathrm{f}}}\left(i \lambda+m_{f}\right) \quad(3.14) \\
& \times \frac{\int \mathrm{d} B \exp \left[\frac{\alpha_{2}}{2}(\operatorname{Tr} B-2 i k)^{2}-\frac{N}{2} \operatorname{Tr} B^{2}\right] \prod_{f=1}^{2 N_{\mathrm{f}}+2} \operatorname{det}\left[i B+\left(m_{f}+i \lambda^{\prime}\right) \mathbb{1}_{N-1}\right]}{\int \mathrm{d} A \exp \left[\frac{\alpha_{2}}{2}(\operatorname{Tr} A-2 i k)^{2}-\frac{N}{2} \operatorname{Tr} A^{2}\right] \prod_{f=1}^{2 N_{\mathrm{f}}} \operatorname{det}\left[i A+m_{f} \mathbb{1}_{N}\right]} .
\end{aligned}
$$




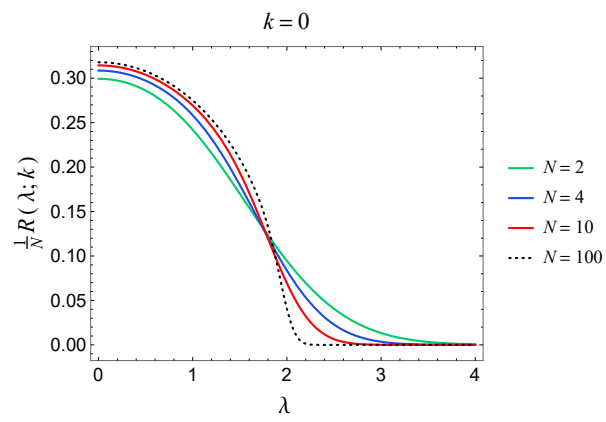

Figure 4. Quenched spectral density at $k=0$. It is an even function of $\lambda$.

\subsection{Quenched limit at finite $N$}

In the quenched limit $N_{\mathrm{f}}=0$, equation (3.10) reduces to

$$
R(\lambda ; k) \sim \mathrm{e}^{-\frac{N}{2} \lambda^{2}} \int_{-\infty}^{\infty} \mathrm{d} x \mathrm{e}^{-N(x-2 i k)(x-\lambda)}\left[H_{N}^{2}\left(\sqrt{\frac{N}{2}} x\right)-H_{N+1}\left(\sqrt{\frac{N}{2}} x\right) H_{N-1}\left(\sqrt{\frac{N}{2}} x\right)\right]
$$

after shifting $x \rightarrow x-\lambda$. Using the orthogonality relations for the Hermite polynomials, the normalization can be easily evaluated. The $x$ integral may also be performed with the help of the formula [85, section 7.374 , eq. 9], leading to the result

$$
\begin{aligned}
R(\lambda ; k)= & \frac{N !}{2^{N+1}} \sqrt{\frac{N}{\pi}} \mathrm{e}^{N\left(\frac{\lambda}{2}-i k\right)^{2}-\frac{N}{2} \lambda^{2}+2 N k^{2}} \\
& \times \sum_{\ell=1}^{N} \frac{2^{2 \ell-N}}{(\ell-1) !(N+1-\ell) !(N-\ell) !} H_{2 N-2 \ell}\left(\frac{\sqrt{N}}{2}(\lambda+2 i k)\right) .
\end{aligned}
$$

Since $H_{2 N-2 k}$ is an even function, $R(\lambda ; k)=R(-\lambda ;-k)$, and one can assume $k \geq 0$ without loss of generality.

As can be seen from figure 4 for the quenched spectral density at $k=0$, in contrast to the standard GUE, the oscillatory structure of the spectral density due to peaks of individual eigenvalues is not present even for small $N$. This feature was also seen in other one-parameter-reweighted ensembles $[72,73]$. We expect that this feature will carry over to three-dimensional QCD as well. This figure also shows that the large $N$-limit, given by the semi-circle $\rho(x)=\sqrt{1-(x / 2)^{2}} / \pi$, is already well-approximated for $N=100$.

When increasing $k$ for a fixed matrix dimension $N$, say $N=20$, the spectral "density" becomes complex-valued. We illustrate this in figure 5 where the real and imaginary parts of the level density $R(\lambda ; k)$ are shown. At nonzero $k>0$, the semi-circle undergoes a dramatic deformation of its shape. First, small oscillations at the two edges appear. They even change the sign of the spectral density for small regions regardless of how small $k$ is. The amplitude of these oscillations grows with $k$. While keeping $k$ below a threshold $k_{c}$, see the ensuing subsections, the oscillations die out around the origin and we can expect a well-defined microscopic limit. Yet, when increasing $k$ beyond $k_{c}$, the oscillations intensify and move into the bulk of the spectrum, see figure 6 , such that even there the spectral 

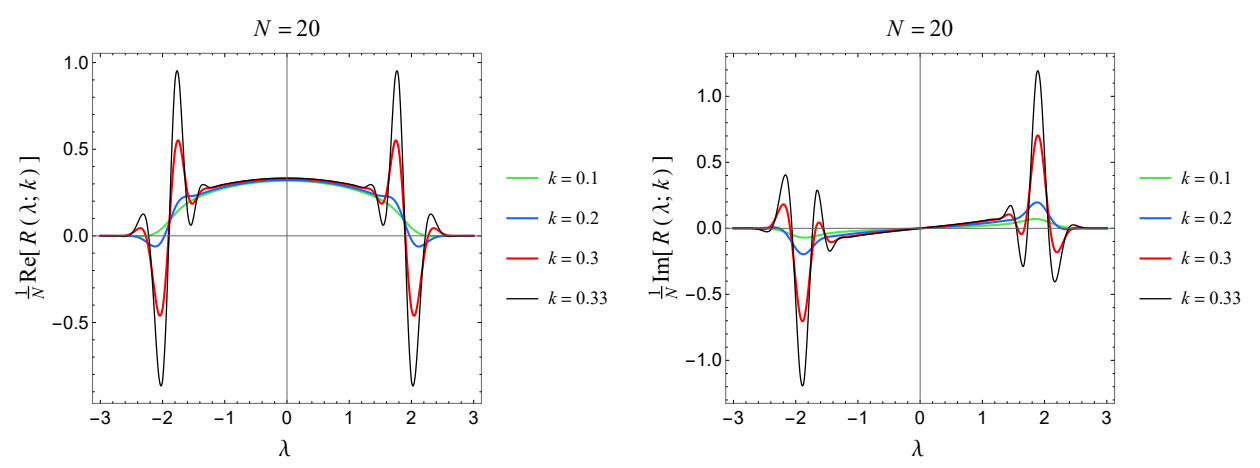

Figure 5. The real (left) and imaginary (right) part of the quenched spectral density for $N=20$.
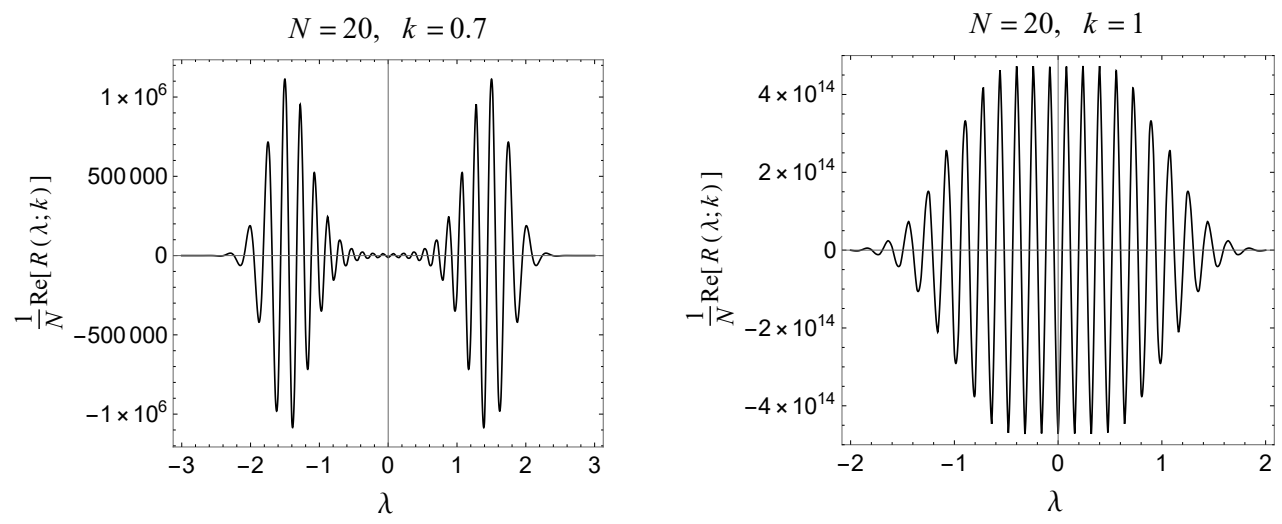

Figure 6. The real part of the quenched spectral density for $N=20$ at $k=0.7$ and 1 .

density does not remain strictly positive. The amplitudes of the oscillations grow rapidly with $k$, even though the normalization condition $\int \mathrm{d} \lambda R(\lambda ; k)=N$ is strictly satisfied. A similar oscillation of the spectral density was also observed in matrix models for QCD at nonzero chemical potential [8, 86-88] and for QCD with nonzero theta angle [89, 90].

The question is how this oscillatory behavior carries over to the large- $N$ limit while keeping $k$ fixed. Three things may happen. Either the oscillations do not reach the origin; then we expect the universal results from GUE with a possible reweighting since the level spacing is changing. Second, the oscillations reach the origin but are not strong enough to make the microscopic limit ill-defined, in particular the amplitude does not grow with the matrix dimension $N$. And third, the oscillations become so dominant that the microscopic limit is not well-defined at the origin. The latter will usually happen at about $k_{c} \approx N_{\mathrm{f}}+1 / 2$ as we will see below.

\subsection{Quenched microscopic large- $N$ limit}

The next task is to evaluate the microscopic limit of the quenched density (3.15) where we evaluate its large- $N$ limit at fixed $\hat{\lambda} \equiv \lambda N$. Incorporating the normalization factor, we 


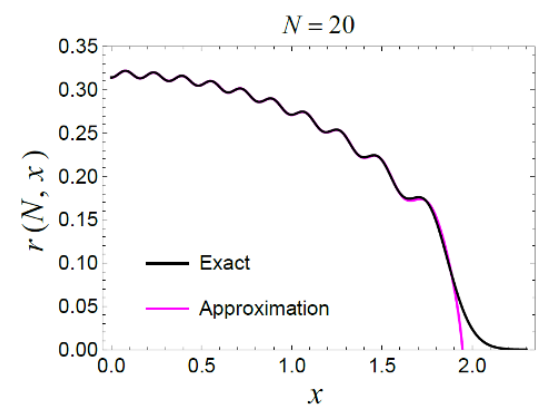

Figure 7. $r(N, x)$ and its large- $N$ approximation (3.21) for $N=20$.

write $(3.15)$ as

$$
\frac{1}{N} R(\lambda ; k)=\frac{\mathcal{N}(N, 0)}{\mathcal{N}(N, k)} \sqrt{\frac{N}{2 \pi}} \mathrm{e}^{-\frac{N}{2} \lambda^{2}} \int_{-\infty}^{\infty} \mathrm{d} x \mathrm{e}^{-N(x-2 i k)(x-\lambda)+\frac{N}{2} x^{2}} r(N, x),
$$

where

$$
\mathcal{N}(N, k)=2^{N+1}(N-1) ! \pi \mathrm{e}^{-2 N k^{2}}
$$

and

$$
r(N, x)=\sqrt{\frac{2 \pi}{N}} \frac{1}{\mathcal{N}(N, 0)} \mathrm{e}^{-\frac{N}{2} x^{2}}\left[H_{N}^{2}\left(\sqrt{\frac{N}{2}} x\right)-H_{N+1}\left(\sqrt{\frac{N}{2}} x\right) H_{N-1}\left(\sqrt{\frac{N}{2}} x\right)\right],
$$

which is normalized as $\int_{-\infty}^{\infty} \mathrm{d} x r(N, x)=1$. While $r(N, x)$ is similar to that of the WignerDyson ensemble, the $N$ dependence is slightly different. However, for large $N$ it also approaches a semi-circle. To obtain more quantitative results we use the uniform asymptotic expansion of the Hermite polynomials [91]

$$
\begin{aligned}
H_{N}(x) \stackrel{N \gg 1}{\approx} \frac{(2 N)^{N / 2} \mathrm{e}^{-N / 2}}{\left(1-\frac{x^{2}}{2 N}\right)^{1 / 4}} \mathrm{e}^{x^{2} / 2} \cos \left[N \left\{\frac{1}{2} \sin \left(2 \arcsin \left(\frac{x}{\sqrt{2 N}}\right)\right)\right.\right. \\
\left.\left.+\arcsin \left(\frac{x}{\sqrt{2 N}}\right)-\frac{\pi}{2}\right\}+\frac{1}{2} \arcsin \left(\frac{x}{\sqrt{2 N}}\right)\right] \\
=\sqrt{2} \frac{(2 N)^{N / 2} \mathrm{e}^{-N / 2}}{\left(1-\frac{x^{2}}{2 N}\right)^{1 / 4}} \mathrm{e}^{x^{2} / 2} \cos \left[\sqrt{N-\frac{x^{2}}{2}} \frac{x}{\sqrt{2}}+\left(N+\frac{1}{2}\right) \arcsin \left(\frac{x}{\sqrt{2 N}}\right)-\frac{N \pi}{2}\right],
\end{aligned}
$$

valid for $|x|<\sqrt{2 N}$. For large $N$, we thus obtain

$$
r(N, x) \stackrel{N \gg 1}{\approx} \frac{1}{\pi} \sqrt{1-\frac{x^{2}}{4}}-\frac{1}{\pi N} \frac{\cos \left[N\left(x \sqrt{1-\frac{x^{2}}{4}}-2 \arccos \frac{x}{2}\right)\right]}{4-x^{2}} .
$$

In figure 7 we compare (3.19) and (3.21) for $N=20$. The agreement is excellent except near the edge of the semi-circle. Returning to the quenched density, it is comprised of two 
pieces

$$
\frac{1}{N} R(\lambda ; k) \stackrel{N \gg 1}{\approx} \frac{1}{N} R^{(a)}(\lambda ; k)+\frac{1}{N} R^{(b)}(\lambda ; k),
$$

associated with the two terms in $(3.21)$, where $R^{(a)}(\lambda ; k)$ corresponds to the semi-circle part and $R^{(b)}(\lambda ; k)$ to the oscillatory part. The prefactor $1 / N$ results from the scale on which we want to zoom in about the origin.

To evaluate the first contribution at large $N$ we note

$$
-N(x-2 i k)(x-\lambda)+\frac{N}{2} x^{2}=N\left(-2 k^{2}+\frac{\lambda^{2}}{2}\right)-\frac{N}{2}(x-2 i k-\lambda)^{2} .
$$

The saddle point can be approximated as $x=2 i k$ since $\lambda=\widehat{\lambda} / N$, so

$$
\frac{1}{N} R^{(a)}(\lambda ; k)=\sqrt{\frac{N}{2 \pi}} \mathrm{e}^{2 N k^{2}-\frac{\widehat{\lambda}^{2}}{2 N}} \int_{-\infty}^{\infty} \mathrm{d} x \mathrm{e}^{-N(x-2 i k)(x-\widehat{\lambda} / N)+\frac{N}{2} x^{2}} \frac{1}{\pi} \sqrt{1-\frac{x^{2}}{4}} \stackrel{N \gg 1}{\approx} \frac{\sqrt{1+k^{2}}}{\pi} .
$$

The average over the oscillatory part can be evaluated as

$$
\begin{aligned}
\frac{1}{N} R^{(b)}(\lambda ; k)= & -\frac{1}{\pi \sqrt{2 \pi N}} \mathrm{e}^{2 N k^{2}-\frac{\widehat{\lambda}^{2}}{2 N}} \int_{-2}^{2} \mathrm{~d} x \mathrm{e}^{-N(x-2 i k)(x-\widehat{\lambda} / N)+\frac{N}{2} x^{2}} \\
& \times \frac{\cos \left[N\left(x \sqrt{1-\frac{x^{2}}{4}}-2 \arccos \frac{x}{2}\right)\right]}{4-x^{2}} \\
& \stackrel{N \gg 1}{\approx}-\frac{1}{2 \pi \sqrt{2 \pi N}} \mathrm{e}^{2 N k^{2}} \int_{-2}^{2} \mathrm{~d} x \frac{\exp \left[N f_{+}(x)\right]+\exp \left[N f_{-}(x)\right]}{4-x^{2}} \mathrm{e}^{(x-2 i k) \widehat{\lambda}} .
\end{aligned}
$$

We have dropped the term $\exp \left[-N \lambda^{2} / 2\right]$ since $\lambda \sim 1 / N$ in the microscopic large- $N$ limit. Furthermore we introduced the function

$$
f_{ \pm}(x)=-(x-2 i k) x+\frac{x^{2}}{2} \pm i\left(x \sqrt{1-\frac{x^{2}}{4}}-2 \arccos \frac{x}{2}\right) .
$$

For large $N$ the integral can be evaluated with the saddle point method. Solving

$$
f_{ \pm}^{\prime}(x)=-x+2 i k \pm 2 i \sqrt{1-\frac{x^{2}}{4}}=0
$$

yields the solutions

$$
\left\{\begin{array}{l}
f_{+}^{\prime}\left(x_{c}\right)=0, \quad \text { for } k<0, \\
f_{-}^{\prime}\left(x_{c}\right)=0, \quad \text { for } k>0,
\end{array} \quad \text { with } \quad x_{c}=i \frac{k^{2}-1}{k} .\right.
$$

To check this, we want to point out that

$$
\sqrt{-\left(\frac{y}{2}-\frac{2}{y}\right)^{2}}=-i \operatorname{sign}\left(\operatorname{Im}\left[\frac{y}{2}-\frac{2}{y}\right]\right)\left(\frac{y}{2}-\frac{2}{y}\right) \text { for } y \notin \mathbb{R}
$$

which forbids a saddle point of $f_{+}(x)$ for $k>0$ and of $f_{-}(x)$ for $k<0$. 


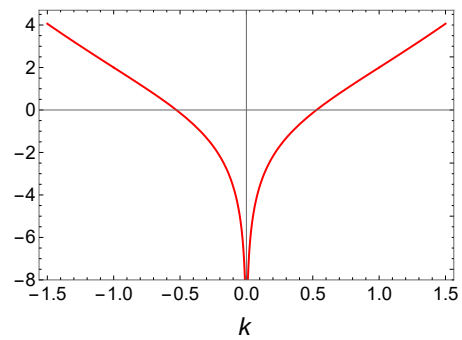

Figure 8. Shown is the function $2 k^{2}+\operatorname{Re}\left[f_{-\operatorname{sign}(k)}\left(x_{c}\right)\right]$.

The saddle point expansion leads to

$$
\begin{aligned}
\frac{1}{N} R^{(b)}(\lambda ; k) \stackrel{N \gg 1}{\approx} & -\frac{1}{2 \pi \sqrt{2 \pi N}} \frac{k^{2}}{\left(k^{2}+1\right)^{2}} \exp \left[N\left(2 k^{2}+f_{-\operatorname{sign}(k)}\left(x_{c}\right)\right)-i \frac{k^{2}+1}{k} \widehat{\lambda}\right] \\
& \times \int_{-\infty}^{\infty} \mathrm{d} x \exp \left[-\frac{N}{1+k^{2}}\left(x-x_{c}\right)^{2}\right] \\
= & -\frac{1}{\sqrt{8} \pi N} \frac{k^{2}}{\left(k^{2}+1\right)^{3 / 2}} \exp \left[N\left(2 k^{2}+f_{-\operatorname{sign}(k)}\left(x_{c}\right)\right)-i \frac{k^{2}+1}{k} \hat{\lambda}\right] .
\end{aligned}
$$

The explicit form of the function $f_{-\operatorname{sign}(k)}\left(x_{c}\right)$ is given by

$$
f_{-\operatorname{sign}(k)}\left(x_{c}\right)=1-k^{2}+2 \operatorname{arcsinh}\left(\frac{k^{2}-1}{2|k|}\right)+\operatorname{sign}(k) \pi i .
$$

To derive this intermediate result we used the identity

$$
\arccos (i x)=\frac{\pi}{2}-i \operatorname{arcsinh}(x) \text { for } x \in \mathbb{R} .
$$

Let us collect all results, so that the microscopic level density reads

$$
\begin{aligned}
\frac{1}{N} R(\lambda ; k) \stackrel{N \gg 1}{\approx} & \frac{\sqrt{1+k^{2}}}{\pi}-\frac{(-1)^{N}}{\sqrt{8} \pi N} \frac{k^{2}}{\left(k^{2}+1\right)^{3 / 2}} \\
& \times \exp \left[N\left(1+k^{2}+2 \operatorname{arcsinh}\left(\frac{k^{2}-1}{2|k|}\right)\right)-i \frac{k^{2}+1}{k} \widehat{\lambda}\right] .
\end{aligned}
$$

This indicates that the amplitude of the oscillation is controlled by the term $1+k^{2}+$ $2 \operatorname{arcsinh}\left[\left(k^{2}-1\right) / 2|k|\right]$, which is shown in figure 8. The function changes sign at $k= \pm k_{c}$ with

$$
k_{c}=\int_{0}^{\infty} \Theta\left[2 \operatorname{arcsinh}\left(\frac{1-k^{2}}{2|k|}\right)-k^{2}-1\right] \mathrm{d} k=0.527697 \cdots,
$$

where $\Theta$ is the Heaviside step function. Thus, for $N \gg 1$, the amplitude of the oscillations grows exponentially for $|k|>k_{c}$, but it dies out for $|k|<k_{c}$. In the limit $k \rightarrow 0$ we smoothly recover the well-known microscopic spectral density of GUE, $\rho_{\text {mic }}(\widehat{\lambda})=1 / \pi$, see [36]. It is intriguing to note that the value of $k_{c}$ in eq. (3.35) coincides with the phase transition point obtained from (2.35) in the limit $N_{\mathrm{f}} \rightarrow+0$. This observation is not surprising when considering an alternative derivation given in the appendix A.3. 

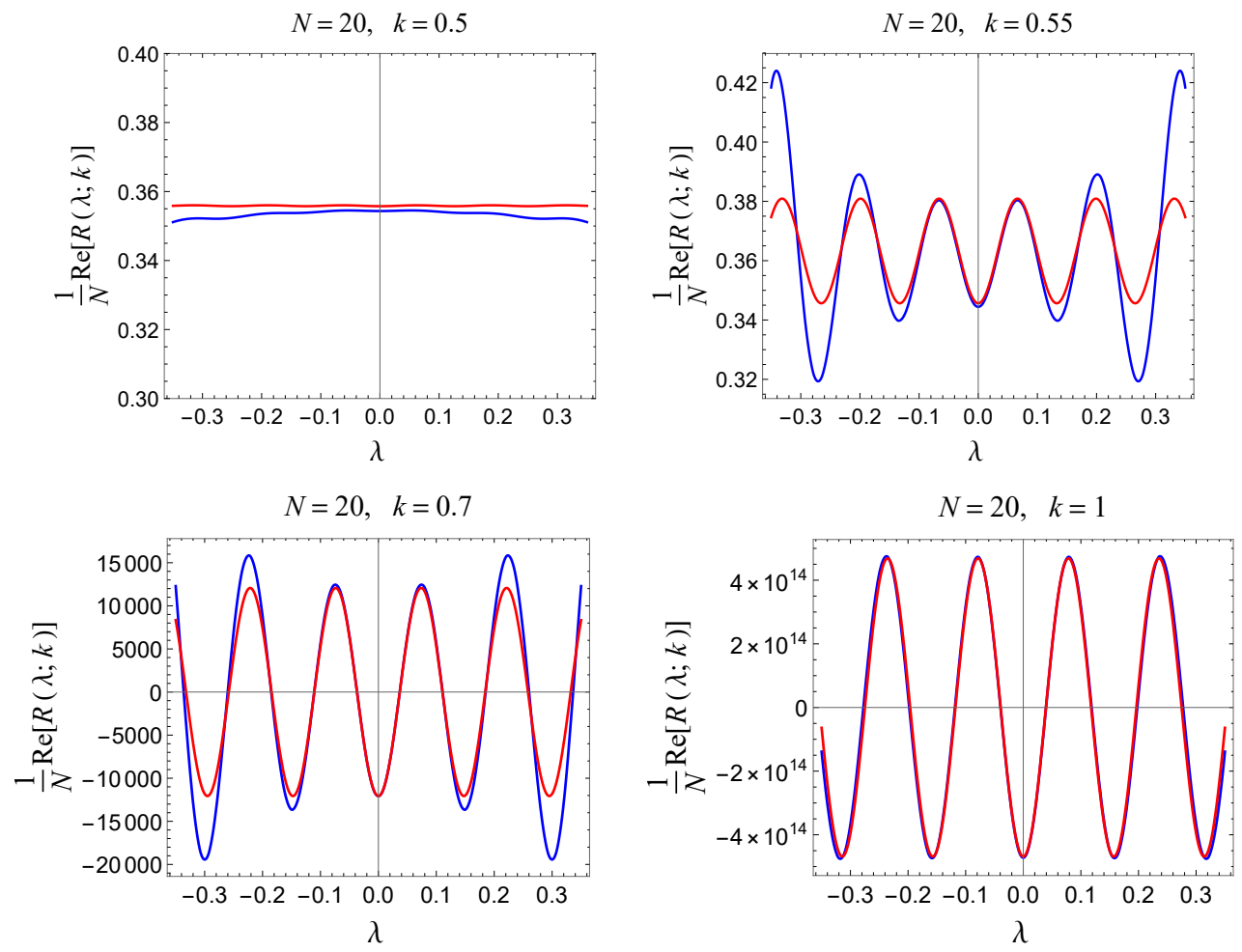

Figure 9. Comparison of the real parts of the exact quenched density (3.16) (blue line) and its asymptotic approximation (3.34) (red line).

In figure 9 we numerically compare (3.34) with the exact density (3.16) for various $k$. In all cases they show good agreement in the region $\lambda \sim 1 / N$ despite the relatively small matrix dimension $N=20$. When increasing $N$ for $|k|>k_{c}$ the oscillations become dominant; the amplitude grows exponentially, and a microscopic limit does not exist.

The quark-antiquark condensate in the quenched case can be readily calculated since the microscopic spectral density is $\widehat{R}(\widehat{\lambda} ; k)=\sqrt{1+k^{2}} / \pi$. Hence, the quark-antiquark condensate is equal to

$$
\Sigma V=\int_{-\infty}^{\infty} \frac{\widehat{R}(\widehat{\lambda} ; k)}{i \widehat{\lambda}+\widehat{m}} d \widehat{\lambda}=\operatorname{sign}(\widehat{m}) \sqrt{1+k^{2}}
$$

This result only holds for $|k|<k_{c}$.

\subsection{Unquenched microscopic level density}

To derive the microscopic level density with dynamical quarks, we start from eq. (3.14) where we have to compute two kinds of partition functions. For this purpose, we first need 
to approximate the prefactor in eq. (3.14) which in the microscopic limit simplifies to

$$
\begin{aligned}
& (-1)^{N-1} \frac{\pi^{N-1}}{(N-1) !} \exp \left[-\frac{\left(2 N_{\mathrm{f}}+1\right) N}{4\left(N_{\mathrm{f}}+1\right)} \lambda^{2}-i \frac{N}{N_{\mathrm{f}}+1} k \lambda\right] \prod_{f=1}^{2 N_{\mathrm{f}}}\left(i \lambda+m_{f}\right) \\
& \stackrel{N \gg 1}{\approx}(-1)^{N-1} \frac{\pi^{N-3 / 2} e^{N}}{\sqrt{2} N^{N-1 / 2+2 N_{\mathrm{f}}}} \mathrm{e}^{-i \frac{k \widehat{\lambda}}{N_{\mathrm{f}}+1}} \prod_{f=1}^{2 N_{\mathrm{f}}}\left(i \widehat{\lambda}+\widehat{m}_{f}\right) .
\end{aligned}
$$

We recall that in this limit $\lambda N \equiv \widehat{\lambda}$ and $m_{f} N \equiv \widehat{m}_{f}$ with $\widehat{\lambda}$ and $\widehat{m}_{f}$ fixed in the limit $N \rightarrow \infty$. The two partition functions in the numerator and denominator of (3.14) are computed in detail in the appendix A with the aid of random matrix methods.

Let us briefly revisit the quenched density. To obtain this quantity we combine eqs. (A.3), (A.30) and (3.37), the latter two for $N_{\mathrm{f}}=0$, in (3.14). Since the partition function $Z_{N_{\mathrm{f}}=0}=Z_{\mathrm{q}}$ is always in the trivial phase $k_{L}=0$ for $|k|<k_{c} \approx 0.527697$ and $\widehat{\widetilde{M}}=-i \widehat{\lambda} / 2 \mathbb{1}_{2}$, we obtain

$$
\frac{1}{N} R(\lambda ; M ; k) \stackrel{N \gg 1}{\approx} \frac{\exp \left[2 N k^{2}-N\left(\lambda_{+}^{2}+\lambda_{-}^{2}\right) / 2+N\right]}{\pi} \sqrt{1+k^{2}} \exp \left[i \frac{\lambda_{+}+\lambda_{-}-2 k}{2} \widehat{\lambda}\right] .
$$

Since $\lambda_{ \pm}=k \pm \sqrt{1+k^{2}}$ in the present case (see eq. (A.12) for the definition for arbitrary $N_{f}$ ), we can simplify this expression to

$$
\frac{1}{N} R(\lambda ; M ; k) \stackrel{N \gg 1}{\approx} \frac{\sqrt{1+k^{2}}}{\pi} .
$$

This is the leading order term in eq. (3.34). The oscillatory part which becomes dominant for $|k|>k_{c}$ can be obtained from eq. (A.30) in the limit $k_{L} \rightarrow 1$ instead of $k_{L}=0$. The limit is important since some terms seem to diverge; nevertheless they cancel with other terms that vanish so that one needs to employ l'Hospital's rule several times.

In the case of dynamical quarks, we collect the terms in eqs. (A.18), (A.30) and (3.37) and find

$$
\begin{aligned}
\frac{1}{N} R(\lambda ; M ; k) \stackrel{N \gg 1}{\approx} \frac{(-1)^{k_{L}}}{2 \pi} \frac{\left(N_{\mathrm{f}}+k_{L}\right) !\left(N_{\mathrm{f}}-k_{L}\right) !}{\left(2 N_{\mathrm{f}}+1\right) !\left(2 N_{\mathrm{f}}\right) !}\left(\frac{\sqrt{\left(N_{\mathrm{f}}+1\right)^{2}+k^{2}-k_{L}^{2}}-k}{\sqrt{\left(N_{\mathrm{f}}+1\right)^{2}+k^{2}-k_{L}^{2}}+k} \frac{N_{\mathrm{f}}+1+k_{L}}{N_{\mathrm{f}}+1-k_{L}}\right)^{2 k_{L}} \\
\quad \times\left(2 \frac{\left(N_{\mathrm{f}}+1\right) \sqrt{\left(N_{\mathrm{f}}+1\right)^{2}+k^{2}-k_{L}^{2}}-k k_{L}}{\left(N_{\mathrm{f}}+1\right)^{2}-k_{L}^{2}}\right)^{2 N_{\mathrm{f}}+1} \mathrm{e}^{-i\left(\lambda_{+}+\lambda_{-}\right) \widehat{\lambda}} \prod_{f=1}^{2 N_{\mathrm{f}}}\left(\widehat{\lambda}-i \widehat{m}_{f}\right) \\
\quad \times \frac{\int_{\mathrm{U}\left(2 N_{\mathrm{f}}+2\right)} \mathrm{d} \mu(U) \mathrm{e}^{-\operatorname{Tr} U^{\dagger} \operatorname{diag}\left(\lambda_{-} \mathbb{1}_{N_{\mathrm{f}}+1+k_{L}}, \lambda_{+} \mathbb{1}_{N_{\mathrm{f}}+1-k_{L}}\right) U \operatorname{diag}\left(\widehat{m}_{1}, \ldots, \widehat{m}_{2 N_{\mathrm{f}}},-i \widehat{\lambda},-i \widehat{\lambda}\right)}}{\int_{\mathrm{U}\left(2 N_{\mathrm{f}}\right)} \mathrm{d} \mu(U) \mathrm{e}^{-\operatorname{Tr} U^{\dagger} \operatorname{diag}\left(\lambda_{-} \mathbb{1}_{N_{\mathrm{f}}+k_{L}}, \lambda_{+} \mathbb{1}_{N_{\mathrm{f}}-k_{L}}\right) U \operatorname{diag}\left(\widehat{m}_{1}, \ldots, \widehat{m}_{2 N_{\mathrm{f}}}\right)}} .
\end{aligned}
$$

Now, we combine the saddle point solutions $\lambda_{ \pm}$into

$$
\omega=\frac{\lambda_{+}-\lambda_{-}}{2}=\frac{\left(N_{\mathrm{f}}+1\right) \sqrt{\left(N_{\mathrm{f}}+1\right)^{2}+k^{2}-k_{L}^{2}}-k k_{L}}{\left(N_{\mathrm{f}}+1\right)^{2}-k_{L}^{2}}
$$


and their sum, and we perform the two Harish-Chandra-Itzykson-Zuber integrals [82, 83] in (3.40) which yields the simplification

$$
\begin{aligned}
& \frac{1}{N} R(\lambda ; M ; k) \stackrel{N \gtrsim 1}{\approx} \frac{(-1)^{k_{L}+1}}{2 \pi}\left(\frac{\sqrt{\left(N_{\mathrm{f}}+1\right)^{2}+k^{2}-k_{L}^{2}}-k}{\sqrt{\left(N_{\mathrm{f}}+1\right)^{2}+k^{2}-k_{L}^{2}}+k} \frac{N_{\mathrm{f}}+1+k_{L}}{N_{\mathrm{f}}+1-k_{L}}\right)^{2 k_{L}} \omega
\end{aligned}
$$

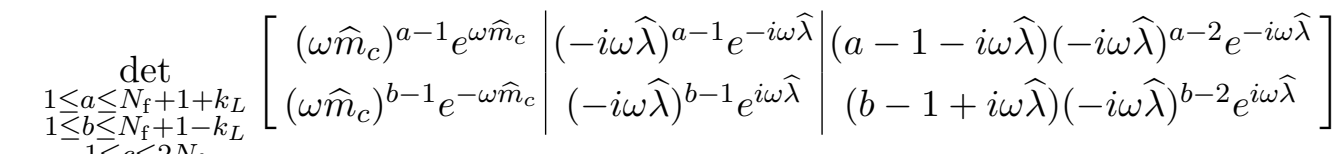

$$
\begin{aligned}
& \prod_{f=1}^{2 N_{\mathrm{f}}}\left(\omega \widehat{\lambda}-i \omega \widehat{m}_{f}\right) \operatorname{det}_{\substack{1 \leq a \leq N_{\mathrm{f}}+k_{L} \\
1 \leq b \leq N_{\mathrm{f}}-k_{L} \\
1 \leq c \leq 2 N_{\mathrm{f}}}}\left[\begin{array}{c}
\left(\omega \widehat{m}_{c}\right)^{a-1} e^{\omega \widehat{m}_{c}} \\
\left(\omega \widehat{m}_{c}\right)^{b-1} e^{-\omega \widehat{m}_{c}}
\end{array}\right]
\end{aligned}
$$

Thence, the density for $k_{L}=0$ is essentially the one with no Chern-Simons term. Interestingly, at the phase transition points the sign of the level density jumps. Especially in the case of $k$ being an integer, the result reduces to

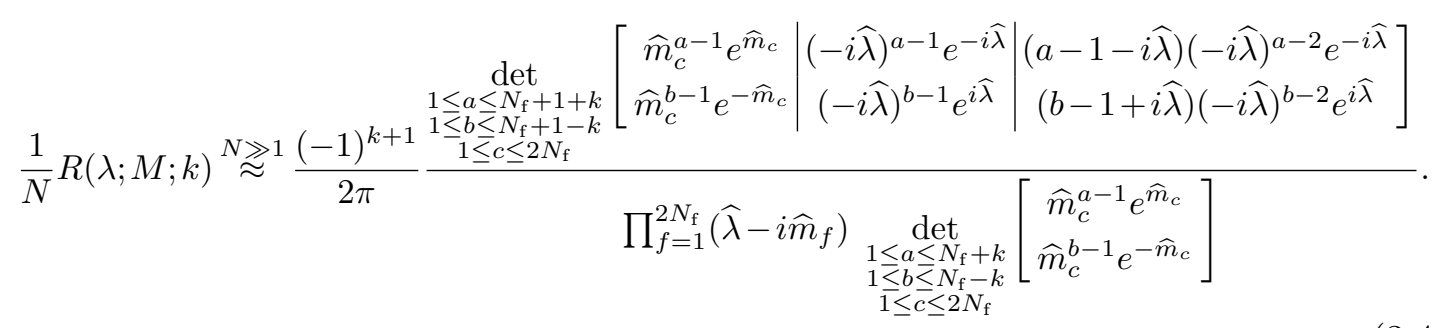

Here, we want to underline as before that we assume that $|k|$ is smaller than a critical value $k_{c}>N_{\mathrm{f}}+1 / 2$. Above this value, the microscopic level density is governed by the oscillations as in the unquenched system, and a microscopic limit does not exist. The corresponding oscillatory part can be obtained by choosing $k_{L}=N_{\mathrm{f}}$ in eq. (A.18) and $k_{L}=N_{\mathrm{f}}+1$ in eq. (A.30). The amplitude will again grow exponentially with $N$. The critical value $k_{c}$ for a fixed number of flavors $2 N_{\mathrm{f}}$ can be obtained from (2.35) by comparing $\lfloor k\rfloor=N_{\mathrm{f}}$ and $\lceil k\rceil=N_{\mathrm{f}}+1$.

Finally we want to present the result for two flavors $\left(2 N_{\mathrm{f}}=2\right)$. For the phase $k_{L}=0$, the level density is equal to

$$
\begin{aligned}
\frac{1}{N} R_{2 N_{\mathrm{f}}=2}\left(\lambda ; m_{1}, m_{2} ; k\right) & \stackrel{N \gg 1}{\approx} \frac{\omega}{\pi}\left(1+\frac{\omega\left(\widehat{m}_{1}-\widehat{m}_{2}\right)}{\sinh \left[\omega\left(\widehat{m}_{1}-\widehat{m}_{2}\right)\right]} \frac{\sinh \left[\omega\left(\widehat{m}_{1}+i \widehat{\lambda}\right)\right] \sinh \left[\omega\left(\widehat{m}_{2}+i \widehat{\lambda}\right)\right]}{\omega^{2}\left(\widehat{\lambda}-i \widehat{m}_{1}\right)\left(\widehat{\lambda}-i \widehat{m}_{2}\right)}\right) \\
& =\omega \rho_{\text {mic }}^{2 N_{\mathrm{f}}=2}\left(\omega \widehat{\lambda} ; \omega \widehat{m}_{1}, \omega \widehat{m}_{2} ; k=0\right)
\end{aligned}
$$

with $\omega=\sqrt{1+k^{2} / 4}$, cf. eq. (3.41), and $\rho_{\text {mic }}^{2 N_{\mathrm{f}}=2}(k=0)$ being the microscopic level density without a Chern-Simons term. Interestingly, the whole spectrum is only rescaled by the factor $\omega$, in particular the mean level spacing is not anymore $\pi$ but $\pi / \omega$. We recall that $|k|$ has to be smaller than the critical value of $0.50551 \ldots$, see table 1 . When $m_{1}=-m_{2}=m$ 

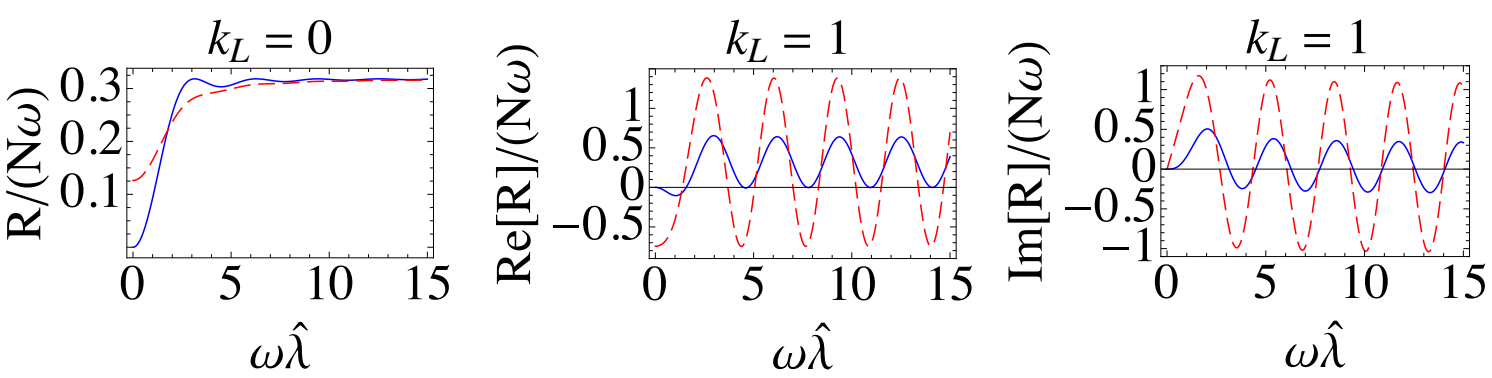

Figure 10. Microscopic level density for two flavors, $2 N_{\mathrm{f}}=2$, at the masses $N m_{1}=-N m_{2}=0$ (blue solid curves) and at $N m_{1}=-N m_{2}=1.5$ (red dashed curves). The left plot shows the level density in the phase $k_{L}=0$, see $(3.45)$, which is the standard situation without or very weak $|k|<0.50551 \ldots$ Chern-Simons term. In particular the level density is real and positive. The middle and right plot represent the real and imaginary part of the microscopic level density in the phase $k_{L}=1$, cf., eq. (3.49). Since only the normalization constant in front of the level density changes with $k$ inside this phase (note the unfolding with $\omega$ ), we have chosen $k=1$. We recall that the imaginary part is an odd function around the origin while the real part is an even function.

the density is evidently real

$$
\begin{aligned}
\frac{1}{N} R_{2 N_{\mathrm{f}}=2}\left(\lambda ; m_{1}=-m_{2}=m ; k\right) & \stackrel{N \gg 1}{\approx} \frac{\omega}{\pi}\left(1-\frac{2 \omega \widehat{m}}{\sinh [2 \omega \widehat{m}]} \frac{|\sinh [\omega(\widehat{m}+i \widehat{\lambda})]|^{2}}{\omega^{2}\left(\widehat{\lambda}^{2}+\widehat{m}^{2}\right)}\right) \\
& =\omega \rho_{\text {mic }}^{2 N_{\mathrm{f}}=2}(\omega \widehat{\lambda} ; \omega \widehat{m} ; 0) .
\end{aligned}
$$

It is shown in the left plot of figure 10 for two different quark masses. When taking the masses to infinity $\widehat{m} \rightarrow \infty$ the quarks decouple and we recover the quenched case $\rho_{\text {mic }}(\widehat{\lambda})=1 / \pi$ as expected.

The corresponding quark-antiquark condensates for $\widehat{m}=\widehat{m}_{1}=\widehat{m}_{2}$ and $\widehat{m}=\widehat{m}_{1}=$ $-\widehat{m}_{2}$ are in this phase equal to

$$
\Sigma V=\frac{1}{2} \frac{\partial}{\partial \widehat{m}} \log Z_{2 N_{\mathrm{f}}=2}^{\beta=2}\left(\widehat{m}, \widehat{m} ; k, k_{L}=0\right)=\frac{1}{2} \frac{\partial}{\partial \widehat{m}} \log \left[e^{-\left(\lambda_{+}+\lambda_{-}\right)} \widehat{m}\right]=-\frac{\lambda_{+}+\lambda_{-}}{2}
$$

and

$$
\Sigma V=\frac{1}{2} \frac{\partial}{\partial \widehat{m}} \log Z_{2 N_{\mathrm{f}}=2}^{\beta=2}\left(\widehat{m},-\widehat{m} ; k, k_{L}=0\right)=\frac{1}{2} \frac{\partial}{\partial \widehat{m}} \log \left[\frac{\sinh [2 \omega \widehat{m}]}{\widehat{m}}\right]=\frac{\omega}{\tanh [2 \omega \widehat{m}]}-\frac{1}{2 \widehat{m}},
$$

respectively.

In the nontrivial phase $k_{L}=1$ (the case $k_{L}=-1$ is very similar), the level density has the form

$$
\begin{array}{r}
\frac{1}{N} R_{2 N_{\mathrm{f}}=2}\left(\lambda ; m_{1}, m_{2} ; k\right) \stackrel{N \gg 1}{\approx} \frac{9 \omega}{\pi}\left(\frac{\sqrt{3+k^{2}}-k}{\sqrt{3+k^{2}}+k}\right)^{2}\left(1+\frac{i}{2}\left[\frac{1}{\omega\left(\widehat{\lambda}-i \widehat{m}_{1}\right)}+\frac{1}{\omega\left(\widehat{\lambda}-i \widehat{m}_{2}\right)}\right]\right. \\
\left.+\frac{e^{-\omega\left(\widehat{m}_{1}+\widehat{m}_{2}\right)-2 i \omega \widehat{\lambda}}}{\omega\left(\widehat{m}_{1}-\widehat{m}_{2}\right)}\left[\frac{\widehat{\lambda}-i \widehat{m}_{1}}{\widehat{\lambda}-i \widehat{m}_{2}} e^{\omega\left(\widehat{m}_{1}-\widehat{m}_{2}\right)}-\frac{\widehat{\lambda}-i \widehat{m}_{2}}{\widehat{\lambda}-i \widehat{m}_{1}} e^{\omega\left(\widehat{m}_{2}-\widehat{m}_{1}\right)}\right]\right)
\end{array}
$$




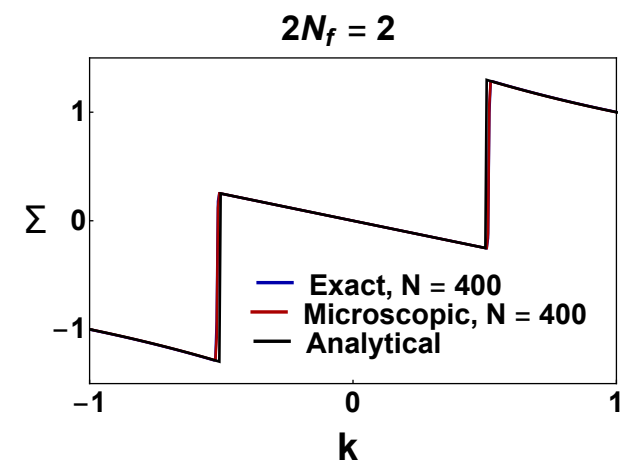

Figure 11. The $k$ dependence of the condensate in the limit of zero quark masses. The exact result (blue curve) and microscopic result (red curve) have been obtained from the partition functions (2.52) and (A.18), for $N=400$ and $m_{1}=m_{2}=10^{-4}$, respectively. Note that the two curves completely overlap. The black curve represents the analytical result (3.51).

with $\omega=\left(2 \sqrt{3+k^{2}}-k\right) / 3$ [cf. eq. (3.41)]. This spectral density has always a non-trivial imaginary part even when we set $m_{1}=-m_{2}=m$ in which case it simplifies to

$$
\begin{array}{r}
\frac{1}{N} R_{2 N_{\mathrm{f}}=2}\left(\lambda ; m_{1}=-m_{2}=m ; k\right) \stackrel{N \gg 1}{\approx} \frac{9 \omega}{\pi}\left(\frac{\sqrt{3+k^{2}}-k}{\sqrt{3+k^{2}}+k}\right)^{2}\left(1+i \frac{\omega \widehat{\lambda}}{\omega^{2}\left(\widehat{\lambda}^{2}+\widehat{m}^{2}\right)}\right. \\
\left.+\frac{\omega^{2}\left(\widehat{\lambda}^{2}-\widehat{m}^{2}\right)}{\omega^{2}\left(\widehat{\lambda}^{2}+\widehat{m}^{2}\right)} \frac{\sinh [2 \omega \widehat{m}]}{2 \omega \widehat{m}} e^{-2 i \omega \widehat{\lambda}}-i \frac{\omega \widehat{\lambda}}{\omega^{2}\left(\widehat{\lambda}^{2}+\widehat{m}^{2}\right)} \cosh [2 \omega \widehat{m}] e^{-2 i \omega \widehat{\lambda}}\right) .
\end{array}
$$

From these results we can read off several things. First of all, the level density exhibits complex oscillations in the phase $k_{L}=1$, cf., middle and right plot in figure 10, which also holds for any $\left|k_{L}\right|>0$ phase when considering even more flavors. Additionally, the amplitude of these oscillations grows exponentially in the quark masses. Therefore, we cannot expect that the quenched limit exists in this phase; especially the reduction of the number of flavors does not work anymore. Finally, the change from one phase to another, say $k_{L} \rightarrow k_{L}+1$, drastically changes the microscopic spectral density. There is not a smooth transition of the microscopic level densities in the various phases and it completely breaks down when $|k|$ crosses a critical $k_{c}$, see discussion above.

The quark-antiquark condensate as a function of $k$ readily follows from the partition function which for $2 N_{\mathrm{f}}$ has three components

$$
Z_{2 N_{\mathrm{f}}=2}^{\beta=2}\left(\widehat{m}_{1}, \widehat{m}_{2} ; k, k_{L}=1\right)+Z_{2 N_{\mathrm{f}}=2}^{\beta=2}\left(\widehat{m}_{1}, \widehat{m}_{2} ; k, k_{L}=0\right)+Z_{2 N_{\mathrm{f}}=2}^{\beta=2}\left(\widehat{m}_{1}, \widehat{m}_{2} ; k, k_{L}=-1\right) .
$$

The explicit expressions are given in eq. (A.18). In the thermodynamic limit only one of the three components contributes to the condensate depending on the value of $k$. For $m_{1}=m_{2}$ the integrand in eq. (A.19) does not depend on $U$ resulting in a pure exponential mass dependence. In the microscopic limit, we thus find a mass independent chiral condensate 
given by

$$
\begin{aligned}
\Sigma(k)= & -\theta\left(k-k_{c}\right) \lambda_{-}\left(k, k_{L}=1\right)-\theta\left(-k-k_{c}\right) \lambda_{+}\left(k, k_{L}=-1\right) \\
& -\frac{1}{2} \theta\left(k_{c}-k\right) \theta\left(k+k_{c}\right)\left(\lambda_{-}\left(k, k_{L}=0\right)+\lambda_{+}\left(k, k_{L}=0\right)\right)
\end{aligned}
$$

with $k_{c}=0.50551$ (see table 1 ). Up to $1 / N$ corrections, this result (black curve in figure 11) is in agreement with the $k$ dependence of the condensate for close to massless quarks obtained from the exact partition function (2.52) (blue curve in figure 11) which coincides with the result from the microscopic partition function (A.18) (red curve in figure 11). The small discrepancy between the last two curves and the analytical result is due to $1 / N$ corrections - taking the quark masses closer to zero does not change the curves.

\section{Conclusion and outlook}

We have constructed a random matrix theory for $\mathrm{QCD}$ in three dimensions $\left(\mathrm{QCD}_{3}\right)$ with a Chern-Simons term of level $k$ that reproduces the pattern of spontaneous symmetry breaking according to $\mathrm{U}\left(2 N_{\mathrm{f}}\right) \rightarrow \mathrm{U}\left(N_{\mathrm{f}}+k\right) \times \mathrm{U}\left(N_{\mathrm{f}}-k\right)$ as proposed recently by Komargodski and Seiberg [48]. This random matrix model is an extension of the random matrix for $\mathrm{QCD}_{3}$ without a Chern-Simons term $(k=0)$. The Chern-Simons term of the random matrix model is in some aspects different in character from the Chern-Simons term of QCD in 3 dimensions but agrees in other aspects. In particular, the level $k$ is not quantized, which is not surprising since the random matrix model does not have a local gauge invariance. However, the effect of the Chern-Simons term on the eigenvalues is similar - it adds a phase proportional to $k$ to the phase of the fermion determinant. It is remarkable that, in all cases we know of, random matrix theories with global symmetries of QCD-like theories reproduce their pattern of spontaneous symmetry breaking and break the symmetry in such a way that the corresponding condensate has the maximum global symmetry, see ref. [92]. The present work shows that a complex action can violate this feature, even in the case of random matrix theory.

What we have learned from earlier work on random matrix theory with a complex action is that the imaginary part of the action can move the phase boundaries of the phase quenched theory. For QCD at nonzero chemical potential the phase of the fermion determinant moves the critical chemical potential of half the pion mass to $1 / 3$ of the baryon mass. For QCD at nonzero theta angle, the chiral condensate does not change sign when one of the quark masses does not change sign. Keeping this in mind, it is not unexpected that the phase due to the Chern-Simons term can change the phase of the theory: the imaginary part of the action nullifies the leading phase so that the subleading phase becomes dominant. At $k \neq 0$, the phase with the standard pattern of chiral symmetry breaking is canceled, so that phases with asymmetric breaking of spontaneous symmetry breaking becomes dominant.

\section{Acknowledgments}

J.V. acknowledges partial support from U.S. DOE Grant No. DE-FAG-88FR40388, and M.K. acknowledges the support by the German research council (DFG) via the CRC 1283: 
"Taming uncertainty and profiting from randomness and low regularity in analysis, stochastics and their applications". Ideas related to this paper are also discussed in [93]. Zohar Komargodski is thanked for discussions and sharing a draft of the paper.

\section{A Derivation of some partition functions}

In this appendix we work out the explicit computation of the two partition functions in eq. (3.14). The one in the denominator has to be dealt separately for the quenched (subsection A.1) and unquenched (subsection A.2) ensemble while this distinction is not relevant for the partition function in the numerator, which is evaluated in subsection A.3.

\section{A.1 Quenched $A$ integral}

We first consider the quenched partition function

$$
Z_{\mathrm{q}}=\int \mathrm{d} A \exp \left[\frac{\alpha_{2}}{2}(\operatorname{Tr} A-2 i k)^{2}-\frac{N}{2} \operatorname{Tr} A^{2}\right],
$$

and linearize the squared term by introducing an auxiliary $x$-integral,

$$
Z_{\mathrm{q}}=\int_{-\infty}^{\infty} \frac{\mathrm{d} x}{\sqrt{2 \alpha_{2} \pi}} \int \mathrm{d} A \exp \left[-\frac{x^{2}}{2 \alpha_{2}}-x(\operatorname{Tr} A-2 i k)-\frac{N}{2} \operatorname{Tr} A^{2}\right]
$$

Next, we shift $A \rightarrow A-x / N \mathbb{1}_{N}$ and integrate over $A$,

$$
Z_{\mathrm{q}}=2^{N / 2}\left(\frac{\pi}{N}\right)^{N^{2} / 2} \int_{-\infty}^{\infty} \frac{\mathrm{d} x}{\sqrt{2 \alpha_{2} \pi}} \exp \left[-\frac{1-\alpha_{2}}{2 \alpha_{2}} x^{2}+2 i k x\right]
$$

Finally, we perform the integration over $x$ and arrive at

$$
Z_{\mathrm{q}}=\frac{2^{N / 2}(\pi / N)^{N^{2} / 2}}{\sqrt{1-\alpha_{2}}} \exp \left[-\frac{2 \alpha_{2}}{1-\alpha_{2}} k^{2}\right] \stackrel{N \gg 1}{\approx} 2^{N / 2} \sqrt{N}(\pi / N)^{N^{2} / 2} \exp \left[-2 N k^{2}\right] .
$$

\section{A.2 Unquenched $A$ integral}

The next quantity we consider is the unquenched partition function

$$
Z_{N_{\mathrm{f}}}=\int \mathrm{d} A \exp \left[\frac{\alpha_{2}}{2}(\operatorname{Tr} A-2 i k)^{2}-\frac{N}{2} \operatorname{Tr} A^{2}\right] \prod_{f=1}^{2 N_{\mathrm{f}}} \operatorname{det}\left[i A+m_{f} \mathbb{1}_{N}\right] .
$$

As in the previous section, we first linearize the squared term with the help of an $x$-integral and then introduce a Gaussian integral over a complex Grassmann valued $N \times 2 N_{\mathrm{f}}$ matrix $V$,

$$
\begin{aligned}
Z_{N_{\mathrm{f}}}= & \int_{-\infty}^{\infty} \frac{\mathrm{d} x}{\sqrt{2 \alpha_{2} \pi}} \int \mathrm{d} A \exp \left[-\frac{x^{2}}{2 \alpha_{2}}-x(\operatorname{Tr} A-2 i k)-\frac{N}{2} \operatorname{Tr} A^{2}\right] \\
& \times \frac{\int \mathrm{d} V \exp \left[-i \operatorname{Tr} A V V^{\dagger}+\operatorname{Tr} V^{\dagger} V M\right]}{\int \mathrm{d} V \exp \left[\operatorname{Tr} V^{\dagger} V\right]}
\end{aligned}
$$


Here, we used the anticommuting property of Grassmann variables. The integral over $A$ can again be performed after the shift $A \rightarrow A-x / N \mathbb{1}_{N}$ yielding

$$
\begin{aligned}
Z_{N_{\mathrm{f}}}= & 2^{N / 2}\left(\frac{\pi}{N}\right)^{N^{2} / 2} \int_{-\infty}^{\infty} \frac{\mathrm{d} x}{\sqrt{2 \alpha_{2} \pi}} \exp \left[-\frac{1-\alpha_{2}}{2 \alpha_{2}} x^{2}+2 i k x\right] \\
& \times \frac{\int \mathrm{d} V \exp \left[\frac{1}{2 N} \operatorname{Tr}\left(V^{\dagger} V\right)^{2}+\operatorname{Tr} V^{\dagger} V\left(M-i \frac{x}{N} \mathbb{1}_{2 N_{\mathrm{f}}}\right)\right]}{\int \mathrm{d} V \exp \left[\operatorname{Tr} V^{\dagger} V\right]} .
\end{aligned}
$$

In the next stage, we integrate over $x$ and find

$$
Z_{N_{\mathrm{f}}}=\frac{2^{N / 2}(\pi / N)^{N^{2} / 2}}{\sqrt{1-\alpha_{2}}} \frac{\int \mathrm{d} V \exp \left[\frac{1}{2 N} \operatorname{Tr}\left(V^{\dagger} V\right)^{2}+\operatorname{Tr} V^{\dagger} V M-\frac{N}{2\left(2 N_{\mathrm{f}}+1\right)}\left(2 k-\frac{1}{N} \operatorname{Tr} V^{\dagger} V\right)^{2}\right]}{\int \mathrm{d} V \exp \left[\operatorname{Tr} V^{\dagger} V\right]} .
$$

Now we are ready to apply the bosonization formula [94-96] and replace $V^{\dagger} V$ by $N \widetilde{U}$ with $\widetilde{U} \in \mathrm{U}\left(2 N_{\mathrm{f}}\right)$. The scaling factor $N$ is chosen for convenience of the saddle point analysis. Thus, we have

$$
Z_{N_{\mathrm{f}}}=\frac{2^{N / 2}(\pi / N)^{N^{2} / 2}}{\sqrt{1-\alpha_{2}}} \frac{\int \mathrm{d} \mu(\widetilde{U}) \operatorname{det}^{-N} \widetilde{U} \exp \left[\frac{N}{2} \operatorname{Tr} \widetilde{U}^{2}+\operatorname{Tr} \widetilde{U} \widehat{M}-\frac{N}{2\left(2 N_{\mathrm{f}}+1\right)}(2 k-\operatorname{Tr} \widetilde{U})^{2}\right]}{\int \mathrm{d} \mu(\widetilde{U}) \operatorname{det}^{-N} \widetilde{U} \exp [N \operatorname{Tr} \widetilde{U}]},
$$

where $\widehat{M}=N M$ is fixed in the microscopic limit. Next we diagonalize the matrix $\widetilde{U}=$ $U^{\dagger} z U$ with $z$ a diagonal matrix of complex phases,

$$
\begin{aligned}
Z_{N_{\mathrm{f}}}= & \frac{2^{N / 2}(\pi / N)^{N^{2} / 2}}{\sqrt{1-\alpha_{2}}} \frac{\int \frac{\mathrm{d} z}{\operatorname{det}^{N+1} z}\left|\Delta_{2 N_{\mathrm{f}}}(z)\right|^{2} \int \mathrm{d} \mu(U) \exp \left[\frac{N}{2} \operatorname{Tr} z^{2}-\frac{N}{2\left(2 N_{\mathrm{f}}+1\right)}(2 k-\operatorname{Tr} z)^{2}+\operatorname{Tr} U^{\dagger} z U \widehat{M}\right]}{\int \frac{\mathrm{d} z}{\operatorname{det}^{N+1} z}\left|\Delta_{2 N_{\mathrm{f}}}(z)\right|^{2} \exp [N \operatorname{Tr} z]} \\
= & \frac{2^{N / 2}(\pi / N)^{N^{2} / 2}}{(2 \pi i)^{2 N_{\mathrm{f}} N^{2 N_{\mathrm{f}} N}\left(2 N_{\mathrm{f}}\right) ! \sqrt{1-\alpha_{2}}}} \prod_{j=0}^{2 N_{\mathrm{f}}-1} \frac{(N+j) !}{j !} \\
& \times \int \frac{\mathrm{d} z}{\operatorname{det}^{N+1} z}\left|\Delta_{2 N_{\mathrm{f}}}(z)\right|^{2} \int \mathrm{d} \mu(U) \exp \left[\frac{N}{2} \operatorname{Tr} z^{2}-\frac{N}{2\left(2 N_{\mathrm{f}}+1\right)}(2 k-\operatorname{Tr} z)^{2}+\operatorname{Tr} U^{\dagger} z U \widehat{M}\right] .
\end{aligned}
$$

Let $z_{k}$ be one saddle point solution of the saddle point equation

$$
z-z^{-1}+\frac{1}{2 N_{\mathrm{f}}+1}(2 k-\operatorname{Tr} z) \mathbb{1}_{2 N_{\mathrm{f}}}=0
$$

and maximizing the integrand. The relation to $\Lambda_{k}$, discussed in section 2.4, is $z_{k}=\Lambda_{k}^{-1}$ which indeed yields the saddle point equation (2.26) after plugging this relation into (A.11). Therefore, we already know that there are $\left(2 N_{\mathrm{f}}\right) ! /\left[\left(N_{\mathrm{f}}+k_{L}\right) !\left(N_{\mathrm{f}}-k_{L}\right) !\right]$ points satisfying these two conditions, where $k_{L}$ is either the integer above $k$ or below $k$ depending on the phase the system is in. In particular we can choose $z_{k}=\operatorname{diag}\left(\lambda_{+}^{-1} \mathbb{1}_{N_{\mathrm{f}}+k}, \lambda_{-}^{-1} \mathbb{1}_{N_{\mathrm{f}}-k}\right)$ with

$$
\lambda_{ \pm}=\frac{k \pm \sqrt{\left(N_{\mathrm{f}}+1\right)^{2}+k^{2}-k_{L}^{2}}}{N_{\mathrm{f}}+1 \pm k_{L}} .
$$

We underline that these solutions are always real because $k^{2}-k_{L}^{2} \geq-\left|k+k_{L}\right| / 2 \geq-\lceil|k|\rceil \geq$ $-N_{\mathrm{f}}$. Moreover we have always $\lambda_{+}>0$ and $\lambda_{-}<0$. 
The contribution from the fluctuations about the saddle point can be obtained from the expansion $z=z_{k}+i \operatorname{diag}\left(\delta z_{+},-\delta z_{-}\right) / \sqrt{N}$ with $\delta z_{+} \in \mathbb{R}^{N_{\mathrm{f}}+k_{L}}$ and $\delta z_{-} \in \mathbb{R}^{N_{\mathrm{f}}-k_{L}}$, where the phase pre-factors reflect the direction of the original contour. Then, the measure transforms as follows

$$
\begin{aligned}
& \left|\Delta_{2 N_{\mathrm{f}}}(z)\right|^{2} \frac{\mathrm{d} z}{\operatorname{det} z} \\
& N_{\approx} \frac{\left(\lambda_{+}^{-1}-\lambda_{-}^{-1}\right)^{2\left(N_{\mathrm{f}}^{2}-k_{L}^{2}\right)} \Delta_{N_{\mathrm{f}}+k_{L}}^{2}\left(\delta z_{+}\right) \Delta_{N_{\mathrm{f}}-k_{L}}^{2}\left(\delta z_{-}\right)}{N^{\left(N_{\mathrm{f}}+k_{L}\right)\left(N_{\mathrm{f}}+k_{L}-1\right) / 2+\left(N_{\mathrm{f}}-k_{L}\right)\left(N_{\mathrm{f}}-k_{L}-1\right) / 2}} \frac{(-1)^{k_{L}} \lambda_{+}^{N_{\mathrm{f}}+k_{L}} \lambda_{-}^{N_{\mathrm{f}}-k_{L}} \mathrm{~d} \delta z_{+} \mathrm{d} \delta z_{-}}{N^{N_{\mathrm{f}}}} .
\end{aligned}
$$

Exploiting the identity $\lambda_{+} \lambda_{-}=-1$, one can explicitly write

$$
\begin{aligned}
& \left|\Delta_{2 N_{\mathrm{f}}}(z)\right|^{2} \frac{\mathrm{d} z}{\operatorname{det} z} \stackrel{N \gg 1}{\approx} \frac{(-1)^{N_{\mathrm{f}}}}{N^{N_{\mathrm{f}}^{2}+k_{L}^{2}}}\left(\frac{\sqrt{\left(N_{\mathrm{f}}+1\right)^{2}+k^{2}-k_{L}^{2}}+k}{\sqrt{\left(N_{\mathrm{f}}+1\right)^{2}+k^{2}-k_{L}^{2}}-k} \frac{N_{\mathrm{f}}+1-k_{L}}{N_{\mathrm{f}}+1+k_{L}}\right)^{k_{L}} \\
& \times\left(2 \frac{\left(N_{\mathrm{f}}+1\right) \sqrt{\left(N_{\mathrm{f}}+1\right)^{2}+k^{2}-k_{L}^{2}}-k k_{L}}{\left(N_{\mathrm{f}}+1\right)^{2}-k_{L}^{2}}\right)^{2\left(N_{\mathrm{f}}^{2}-k_{L}^{2}\right)} \Delta_{N_{\mathrm{f}}+k_{L}}^{2}\left(\delta z_{+}\right) \Delta_{N_{\mathrm{f}}-k_{L}}^{2}\left(\delta z_{-}\right) \mathrm{d} \delta z_{+} \mathrm{d} \delta z_{-} .
\end{aligned}
$$

In the next step, we expand the determinant

$$
\begin{aligned}
\operatorname{det}^{-N} z \stackrel{N \gg 1}{\approx} & \lambda_{+}^{N\left(N_{\mathrm{f}}+k_{L}\right)} \lambda_{-}^{N\left(N_{\mathrm{f}}-k_{L}\right)} \\
& \times \exp \left[-\sqrt{N} i\left(\lambda_{+} \operatorname{Tr} \delta z_{+}-\lambda_{-} \operatorname{Tr} \delta z_{-}\right)-\frac{1}{2}\left(\lambda_{+}^{2} \operatorname{Tr} \delta z_{+}^{2}+\lambda_{-}^{2} \operatorname{Tr} \delta z_{-}^{2}\right)\right] \\
= & (-1)^{\left(N_{\mathrm{f}}+k_{L}\right) N}\left(\frac{\sqrt{\left(N_{\mathrm{f}}+1\right)^{2}+k^{2}-k_{L}^{2}}+k}{\sqrt{\left(N_{\mathrm{f}}+1\right)^{2}+k^{2}-k_{L}^{2}}-k} \frac{N_{\mathrm{f}}+1-k_{L}}{N_{\mathrm{f}}+1+k_{L}}\right)^{k_{L} N} \\
& \times \exp \left[-\sqrt{N} i\left(\lambda_{+} \operatorname{Tr} \delta z_{+}-\lambda_{-} \operatorname{Tr} \delta z_{-}\right)-\frac{1}{2}\left(\lambda_{+}^{2} \operatorname{Tr} \delta z_{+}^{2}+\lambda_{-}^{2} \operatorname{Tr} \delta z_{-}^{2}\right)\right]
\end{aligned}
$$

and the exponent

$$
\begin{gathered}
\frac{N}{2} \operatorname{Tr} z^{2}-\frac{N(2 k-\operatorname{Tr} z)^{2}}{2\left(2 N_{\mathrm{f}}+1\right)} \stackrel{N \gg 1}{\gtrsim} \frac{N}{2}\left(\left(N_{\mathrm{f}}+k_{L}\right) \lambda_{-}^{2}+\left(N_{\mathrm{f}}-k_{L}\right) \lambda_{+}^{2}-\left(2 N_{\mathrm{f}}+1\right)\left(\lambda_{+}^{2}+\lambda_{-}^{2}-2\right)\right) \\
+\sqrt{N} i\left(\lambda_{+} \operatorname{Tr} \delta z_{+}-\lambda_{-} \operatorname{Tr} \delta z_{-}\right)-\frac{1}{2}\left(\operatorname{Tr} \delta z_{+}^{2}+\operatorname{Tr} \delta z_{-}^{2}\right)+\frac{\left(\operatorname{Tr} \delta z_{+}-\operatorname{Tr} \delta z_{-}\right)^{2}}{2\left(2 N_{\mathrm{f}}+1\right)}, \quad \text { (A.16) }
\end{gathered}
$$

where we employed the saddle point equation. The mass dependent term is independent of the massive modes $\delta z_{ \pm}$. As it should be, the exponents proportional to $\sqrt{N}$ cancel each other so that we are left with the $\delta z_{ \pm}$integrals that can be computed as follows

$$
\begin{aligned}
& \int \mathrm{d} \delta z_{+} \mathrm{d} \delta z_{-} \Delta_{N_{\mathrm{f}}+k_{L}}^{2}\left(\delta z_{+}\right) \Delta_{N_{\mathrm{f}}-k_{L}}^{2}\left(\delta z_{-}\right) \\
& \times \exp \left[-\frac{1}{2}\left(\left(\lambda_{+}^{2}+1\right) \operatorname{Tr} \delta z_{+}^{2}+\left(\lambda_{-}^{2}+1\right) \operatorname{Tr} \delta z_{-}^{2}\right)+\frac{\left(\operatorname{Tr} \delta z_{+}-\operatorname{Tr} \delta z_{-}\right)^{2}}{2\left(2 N_{\mathrm{f}}+1\right)}\right] \\
& =\int_{-\infty}^{\infty} \frac{\sqrt{2 N_{\mathrm{f}}+1} \mathrm{~d} x}{\sqrt{2 \pi}} \int \mathrm{d} \delta z_{+} \mathrm{d} \delta z_{-} \Delta_{N_{\mathrm{f}}+k_{L}}^{2}\left(\delta z_{+}\right) \Delta_{N_{\mathrm{f}}-k_{L}}^{2}\left(\delta z_{-}\right)
\end{aligned}
$$




$$
\begin{aligned}
& \times \mathrm{e}^{-\frac{2 N_{\mathrm{f}}+1}{2} x^{2}-\frac{1}{2}\left(\left(\lambda_{+}^{2}+1\right) \operatorname{Tr} \delta z_{+}^{2}+\left(\lambda_{-}^{2}+1\right) \operatorname{Tr} \delta z_{-}^{2}\right)+x\left(\operatorname{Tr} \delta z_{+}-\operatorname{Tr} \delta z_{-}\right)} \\
& =\frac{\sqrt{2 N_{\mathrm{f}}+1}}{\left(\lambda_{+}^{2}+1\right)^{\left(N_{\mathrm{f}}+k_{L}\right)^{2} / 2}\left(\lambda_{-}^{2}+1\right)^{\left(N_{\mathrm{f}}-k_{L}\right)^{2} / 2}} \\
& \times \int_{-\infty}^{\infty} \frac{\mathrm{d} x}{\sqrt{2 \pi}} \exp \left[-\left(2 N_{\mathrm{f}}+1-\frac{N_{\mathrm{f}}+k_{L}}{\lambda_{+}^{2}+1}-\frac{N_{\mathrm{f}}-k_{L}}{\lambda_{-}^{2}+1}\right) \frac{x^{2}}{2}\right] \\
& \times \int \mathrm{d} \delta z_{+} \Delta_{N_{\mathrm{f}}+k_{L}}^{2}\left(\delta z_{+}\right) \mathrm{e}^{-\operatorname{Tr} \delta z_{+}^{2} / 2} \int \mathrm{d} \delta z_{-} \Delta_{N_{\mathrm{f}}-k_{L}}^{2}\left(\delta z_{-}\right) \mathrm{e}^{-\operatorname{Tr} \delta z_{-}^{2} / 2} \\
& =\frac{\sqrt{N_{\mathrm{f}}+1 / 2}}{\left(\left(N_{\mathrm{f}}+1\right)^{2}+k^{2}-k_{L}^{2}\right)^{1 / 4}}\left(N_{\mathrm{f}}+k_{L}\right) !\left(\prod_{j=0}^{N_{\mathrm{f}}+k_{L}-1} \sqrt{2 \pi} j !\right)\left(N_{\mathrm{f}}-k_{L}\right) !\left(\prod_{j=0}^{N_{\mathrm{f}}-k_{L}-1} \sqrt{2 \pi} j !\right) \\
& \times\left(\frac{\sqrt{\left(N_{\mathrm{f}}+1\right)^{2}+k^{2}-k_{L}^{2}}+k}{\sqrt{\left(N_{\mathrm{f}}+1\right)^{2}+k^{2}-k_{L}^{2}}-k} \frac{N_{\mathrm{f}}+1-k_{L}}{N_{\mathrm{f}}+1+k_{L}}\right)^{-N_{\mathrm{f}} k_{L}} \\
& \times\left(2 \frac{\left(N_{\mathrm{f}}+1\right) \sqrt{\left(N_{\mathrm{f}}+1\right)^{2}+k^{2}-k_{L}^{2}}-k k_{L}}{\left(N_{\mathrm{f}}+1\right)^{2}-k_{L}^{2}}\right)^{\frac{1}{2}-\left(N_{\mathrm{f}}^{2}+k_{L}^{2}\right)} .
\end{aligned}
$$

Now we are ready to put everything together and apply Sterling's formula. We eventually arrive at

$$
\begin{aligned}
Z_{N_{\mathrm{f}}}{ }^{\gtrsim} & \frac{(-1)^{\left(N_{\mathrm{f}}+k_{L}\right) N_{2}} 2^{(N-1) / 2} \pi^{N^{2} / 2} \mathrm{e}^{\frac{N}{2}\left(k_{L}\left(\lambda_{-}^{2}-\lambda_{+}^{2}\right)-\left(N_{\mathrm{f}}+1\right)\left(\lambda_{+}^{2}+\lambda_{-}^{2}\right)+2\left(2 N_{\mathrm{f}}+1\right)\right)}}{N^{\frac{N^{2}}{2}+k_{L}^{2}-N_{\mathrm{f}}^{2}-\frac{1}{2}}\left(\left(N_{\mathrm{f}}+1\right)^{2}+k^{2}-k_{L}^{2}\right)^{1 / 4}} \\
& \times \frac{\left(\prod_{j=0}^{N_{\mathrm{f}}+k_{L}-1} j !\right)\left(\prod_{j=0}^{N_{\mathrm{f}}-k_{L}-1} j !\right)}{\prod_{j=0}^{2 N_{\mathrm{f}}-1} j !} \\
& \times\left(\frac{\sqrt{\left(N_{\mathrm{f}}+1\right)^{2}+k^{2}-k_{L}^{2}}+k}{\sqrt{\left(N_{\mathrm{f}}+1\right)^{2}+k^{2}-k_{L}^{2}}-k} \frac{N_{\mathrm{f}}+1-k_{L}}{N_{\mathrm{f}}+1+k_{L}}\right)^{k_{L}\left(N-N_{\mathrm{f}}+1\right)} \\
& \times\left(2 \frac{\left(N_{\mathrm{f}}+1\right) \sqrt{\left(N_{\mathrm{f}}+1\right)^{2}+k^{2}-k_{L}^{2}}-k k_{L}}{\left(N_{\mathrm{f}}+1\right)^{2}-k_{L}^{2}}\right)^{N_{\mathrm{f}}^{2}-3 k_{L}^{2}+\frac{1}{2}} \\
& \times \int \mathrm{d} \mu(U) \mathrm{e}^{-\operatorname{Tr} U^{\dagger} \operatorname{diag}\left(\lambda_{-} \mathbb{1}_{N_{\mathrm{f}}+k_{L}}, \lambda_{+} \mathbb{1}_{N_{\mathrm{f}}-k_{L}}\right) U \widehat{M}} .
\end{aligned}
$$

In the case when $k$ is an integer, meaning $k=k_{L}$ and $\lambda_{ \pm}= \pm 1$, this result simplifies drastically to

$$
\begin{aligned}
Z_{N_{\mathrm{f}}} \gtrsim & \frac{N{ }^{\left(N_{\mathrm{f}}+k\right) N^{2}} 2^{\frac{N}{2}+N_{\mathrm{f}}^{2}-3 k^{2}} \pi^{N^{2} / 2} \mathrm{e}^{N_{\mathrm{f}} N}}{N^{\frac{N^{2}}{2}+k^{2}-N_{\mathrm{f}}^{2}-\frac{1}{2}} \sqrt{N_{\mathrm{f}}+1}} \frac{\left(\prod_{j=0}^{N_{\mathrm{f}}+k-1} j !\right)\left(\prod_{j=0}^{N_{\mathrm{f}}-k-1} j !\right)}{\prod_{j=0}^{2 N_{\mathrm{f}}-1} j !} \\
& \times \int \mathrm{d} \mu(U) \mathrm{e}^{\operatorname{Tr} U^{\dagger} \operatorname{diag}\left(\mathbb{1}_{N_{\mathrm{f}}+k},-\mathbb{1}_{N_{\mathrm{f}}-k}\right) U \widehat{M}} .
\end{aligned}
$$

Note that the prefactors that only depend on $N$ and $N_{\mathrm{f}}$ could have been absorbed in the definition of the partition function. We would like to emphasize that the partition function 
for integer as well as non-integer $k$ is real, as can be already seen from its definition, and even positive when omitting the factor $(-1)^{\left(N_{\mathrm{f}}+k\right) N}$. The latter can be readily achieved by choosing an even matrix dimension $N$.

\section{A.3 The $B$ integral}

In this section we evaluate the integral

$$
Y_{N_{\mathrm{f}}}=\int \mathrm{d} B \exp \left[\frac{\alpha_{2}}{2}(\operatorname{Tr} B-2 i k)^{2}-\frac{N}{2} \operatorname{Tr} B^{2}\right] \prod_{f=1}^{2 N_{\mathrm{f}}+2} \operatorname{det}\left[i B+\left(m_{f}+i \lambda^{\prime}\right) \mathbb{1}_{N-1}\right]
$$

in the large- $N$ limit. The computation proceeds along the same lines as for $Z_{N_{\mathrm{f}}}$. However, we cannot easily carry over the entire result for $N \rightarrow N-1$ and $N_{\mathrm{f}} \rightarrow N_{\mathrm{f}}+1$ since the standard deviations do not change in the same way.

Again, we introduce the auxiliary real variable $x$ to linearize the squared trace term and the complex Grassmann valued matrix $V$, albeit now it has the dimension $(N-1) \times\left(2 N_{\mathrm{f}}+2\right)$. Collecting the masses in the diagonal matrix $\widetilde{M}=\operatorname{diag}\left(m_{1}, \ldots, m_{2 N_{\mathrm{f}}+2}\right)+i \lambda^{\prime} \mathbb{1}_{2 N_{\mathrm{f}}+2}$, we find the integral

$$
\begin{aligned}
Y_{N_{\mathrm{f}}}= & 2^{(N-1) / 2}\left(\frac{\pi}{N}\right)^{(N-1)^{2} / 2} \int_{-\infty}^{\infty} \frac{\mathrm{d} x}{\sqrt{2 \alpha_{2} \pi}} \mathrm{e}^{-\frac{N_{\mathrm{f}}+1}{2 N} x^{2}+2 i k x} \\
& \times \frac{\int \mathrm{d} V \exp \left[\frac{1}{2 N} \operatorname{Tr}\left(V^{\dagger} V\right)^{2}+\operatorname{Tr} V^{\dagger} V\left(\widetilde{M}-i \frac{x}{N} \mathbb{1}_{2 N_{\mathrm{f}}+2}\right)\right]}{\int \mathrm{d} V \exp \left[\operatorname{Tr} V^{\dagger} V\right]},
\end{aligned}
$$

after the integration over $B$, which is the counterpart of (A.7). Next, we apply the bosonization formula [94-96] and integrate over the variable $x$ so that we obtain

$$
\begin{aligned}
Y_{N_{\mathrm{f}}}= & \frac{2^{(N-2) / 2}(\pi / N)^{(N-1)^{2} / 2} \sqrt{N+2 N_{\mathrm{f}}+1}}{\sqrt{N_{\mathrm{f}}+1}} \\
& \times \frac{\int \mathrm{d} \mu(\widetilde{U}) \operatorname{det}^{-N+1} \widetilde{U} \exp \left[\frac{N}{2} \operatorname{Tr} \widetilde{U}^{2}+\operatorname{Tr} \widetilde{U} \widetilde{\widetilde{M}}-\frac{N}{4\left(N_{\mathrm{f}}+1\right)}(2 k-\operatorname{Tr} \widetilde{U})^{2}\right]}{\int \mathrm{d} \mu(\widetilde{U}) \operatorname{det}^{-N+1} \widetilde{U} \exp [N \operatorname{Tr} \widetilde{U}]}
\end{aligned}
$$

with $\widehat{\widetilde{M}}=N \widetilde{M}$. When diagonalizing $\widetilde{U}=U^{\dagger} \widetilde{z} U$ with $\widetilde{z}$ a $2 N_{\mathrm{f}}+2$ dimensional diagonal matrix of complex phases we obtain

$$
\begin{aligned}
Y_{N_{\mathrm{f}}}= & \frac{2^{(N-2) / 2}(\pi / N)^{(N-1)^{2} / 2} \sqrt{N+2 N_{\mathrm{f}}+1}}{(2 \pi i)^{2 N_{\mathrm{f}}+2} N^{2\left(N_{\mathrm{f}}+1\right)(N-1)}\left(2 N_{\mathrm{f}}+2\right) ! \sqrt{N_{\mathrm{f}}+1}} \prod_{j=0}^{2 N_{\mathrm{f}}+1} \frac{(N-1+j) !}{j !} \\
& \times \int \frac{\mathrm{d} \widetilde{z}}{\operatorname{det}^{N} \widetilde{z}}\left|\Delta_{2 N_{\mathrm{f}}+2}(\widetilde{z})\right|^{2} \exp \left[\frac{N}{2} \operatorname{Tr} \widetilde{z}^{2}-\frac{N}{4\left(N_{\mathrm{f}}+1\right)}(2 k-\operatorname{Tr} \widetilde{z})^{2}\right] \int \mathrm{d} \mu(U) \mathrm{e}^{\operatorname{Tr} U^{\dagger} \widetilde{z} U \widehat{\widetilde{M}}}
\end{aligned}
$$

We are ready for a saddle point analysis of the $\tilde{z}$ integral whose saddle point equation is

$$
\widetilde{z}-\widetilde{z}^{-1}+\frac{1}{2\left(N_{\mathrm{f}}+1\right)}(2 k-\operatorname{Tr} \widetilde{z}) \mathbb{1}_{2 N_{\mathrm{f}}+2}=0 .
$$


By plugging in the choice $\widetilde{z}_{k}=\operatorname{diag}\left(\lambda_{+}^{-1} \mathbb{1}_{N_{\mathrm{f}}+1+k_{L}}, \lambda_{-}^{-1} \mathbb{1}_{N_{\mathrm{f}}+1-k_{L}}\right)$ with exactly the same $\lambda_{ \pm}$ and $k_{L}$ as in section A.2, one can easily verify that this is a solution. The real part of the two corresponding actions is apart from an $N$ also the same, especially

$$
\begin{aligned}
\operatorname{Re}\left(\frac{N}{2} \operatorname{Tr} \widetilde{z}_{k}^{2}-\frac{N\left(2 k-\operatorname{Tr} \widetilde{z}_{k}\right)^{2}}{4\left(N_{\mathrm{f}}+1\right)}-N \operatorname{Tr} \ln \widetilde{z}_{k}\right) \\
\quad=\operatorname{Re}\left(\frac{N}{2} \operatorname{Tr} z_{k}^{2}-\frac{N\left(2 k-\operatorname{Tr} z_{k}\right)^{2}}{2\left(2 N_{\mathrm{f}}+1\right)}-N \operatorname{Tr} \ln z_{k}\right)+N .
\end{aligned}
$$

Thus, the phase transition points from the original $A$ integral and from the original $B$ integral are the same as they should. If they were not equal, the spectral density would be either exponentially small or exponentially large in $N$. Yet, $Y_{N_{\mathrm{f}}}$, with two more flavors than $Z_{N_{\mathrm{f}}}$, has one additional phase transition compared to $Z_{N_{\mathrm{f}}}$ at about $k_{c} \approx \pm\left(N_{\mathrm{f}}+1 / 2\right)$. This suggests the presence of an additional phase transition in the spectral density as compared to the original partition function. Indeed, we have found in section 3.3 that the limit of the microscopic level density does not exist when $|k|$ is larger than a critical value $k_{c}$. Hence we have to stay always in the regime where the two phases of the partition functions $Y_{N_{\mathrm{f}}}$ and $Z_{N_{\mathrm{f}}}$ agree. When this is not the case the oscillation will become dominant and a microscopic limit does not exist, see the discussions in sections 3.3 and 3.4.

The expansion works exactly the same as before, i.e., $\widetilde{z}=\widetilde{z}_{k}+i \operatorname{diag}\left(\delta \widetilde{z}_{+},-\delta \widetilde{z}_{-}\right) / \sqrt{N}$ with $\delta \widetilde{z}_{+} \in \mathbb{R}^{N_{\mathrm{f}}+1+k_{L}}$ and $\delta \widetilde{z}_{-} \in \mathbb{R}^{N_{\mathrm{f}}+1-k_{L}}$. Thence, we have for the measure

$$
\begin{aligned}
\left|\Delta_{2 N_{\mathrm{f}}+2}(\widetilde{z})\right|^{2} \mathrm{~d} \widetilde{z}^{N \gg 1} & \frac{(-1)^{k_{L}}}{N^{\left(N_{\mathrm{f}}+1\right)^{2}+k_{L}^{2}}}\left(2 \frac{\left(N_{\mathrm{f}}+1\right) \sqrt{\left(N_{\mathrm{f}}+1\right)^{2}+k^{2}-k_{L}^{2}}-k k_{L}}{\left(N_{\mathrm{f}}+1\right)^{2}-k_{L}^{2}}\right)^{2\left(\left(N_{\mathrm{f}}+1\right)^{2}-k_{L}^{2}\right)} \\
& \times \Delta_{N_{\mathrm{f}}+1+k_{L}}^{2}\left(\delta \widetilde{z}_{+}\right) \Delta_{N_{\mathrm{f}}+1-k_{L}}^{2}\left(\delta \widetilde{z}_{-}\right) \mathrm{d} \delta \widetilde{z}_{+} \mathrm{d} \delta \widetilde{z}_{-},
\end{aligned}
$$

for the determinant

$$
\begin{aligned}
\operatorname{det}^{-N} \widetilde{z}^{N \gg 1} & (-1)^{\left(N_{\mathrm{f}}+k_{L}+1\right) N}\left(\frac{\sqrt{\left(N_{\mathrm{f}}+1\right)^{2}+k^{2}-k_{L}^{2}}+k}{\sqrt{\left(N_{\mathrm{f}}+1\right)^{2}+k^{2}-k_{L}^{2}}-k} \frac{N_{\mathrm{f}}+1-k_{L}}{N_{\mathrm{f}}+1+k_{L}}\right)^{k_{L} N} \\
& \times \exp \left[-\sqrt{N} i\left(\lambda_{+} \operatorname{Tr} \delta \widetilde{z}_{+}-\lambda_{-} \operatorname{Tr} \delta \widetilde{z}_{-}\right)-\frac{1}{2}\left(\lambda_{+}^{2} \operatorname{Tr} \delta \widetilde{z}_{+}^{2}+\lambda_{-}^{2} \operatorname{Tr} \delta \widetilde{z}_{-}^{2}\right)\right],
\end{aligned}
$$

and for the exponent

$$
\begin{aligned}
& \frac{N}{2} \operatorname{Tr} \widetilde{z}^{2}- \frac{N(2 k-\operatorname{Tr} \widetilde{z})^{2}}{4\left(N_{\mathrm{f}}+1\right)} \stackrel{N \gg 1}{\approx} \frac{N}{2}\left(\left(N_{\mathrm{f}}+k_{L}\right) \lambda_{-}^{2}+\left(N_{\mathrm{f}}-k_{L}\right) \lambda_{+}^{2}-\left(2 N_{\mathrm{f}}+1\right)\left(\lambda_{+}^{2}+\lambda_{-}^{2}-2\right)+2\right) \\
&+\sqrt{N} i\left(\lambda_{+} \operatorname{Tr} \delta \widetilde{z}_{+}-\lambda_{-} \operatorname{Tr} \delta \widetilde{z}_{-}\right)-\frac{1}{2}\left(\operatorname{Tr} \delta \widetilde{z}_{+}^{2}+\operatorname{Tr} \delta \widetilde{z}_{-}^{2}\right)+\frac{\left(\operatorname{Tr} \delta \widetilde{z}_{+}-\operatorname{Tr} \delta \widetilde{z}_{-}\right)^{2}}{4\left(N_{\mathrm{f}}+1\right)},
\end{aligned}
$$

where we again have used the saddle point equation to simplify the result. The integral over the Gaussian fluctuation $\delta \widetilde{z}_{ \pm}$about the saddle point is given by

$$
\int \mathrm{d} \delta \widetilde{z}_{+} \mathrm{d} \delta \widetilde{z}_{-} \Delta_{N_{\mathrm{f}}+1+k_{L}}^{2}\left(\delta \widetilde{z}_{+}\right) \Delta_{N_{\mathrm{f}}+1-k_{L}}^{2}\left(\delta \widetilde{z}_{-}\right)
$$




$$
\begin{aligned}
\times \exp [ & \left.-\frac{1}{2}\left(\left(\lambda_{+}^{2}+1\right) \operatorname{Tr} \delta \widetilde{z}_{+}^{2}+\left(\lambda_{-}^{2}+1\right) \operatorname{Tr} \delta \widetilde{z}_{-}^{2}\right)+\frac{\left(\operatorname{Tr} \delta \widetilde{z}_{+}-\operatorname{Tr} \delta \widetilde{z}_{-}\right)^{2}}{4\left(N_{\mathrm{f}}+1\right)}\right] \\
= & \frac{\sqrt{N_{\mathrm{f}}+1}}{\left(\left(N_{\mathrm{f}}+1\right)^{2}+k^{2}-k_{L}^{2}\right)^{1 / 4}}\left(N_{\mathrm{f}}+1+k_{L}\right) !\left(\prod_{j=0}^{N_{\mathrm{f}}+k_{L}} \sqrt{2 \pi} j !\right) \\
& \times\left(N_{\mathrm{f}}+1-k_{L}\right) !\left(\prod_{j=0}^{N_{\mathrm{f}}-k_{L}} \sqrt{2 \pi} j !\right) \\
& \times\left(\frac{\sqrt{\left(N_{\mathrm{f}}+1\right)^{2}+k^{2}-k_{L}^{2}}+k}{\sqrt{\left(N_{\mathrm{f}}+1\right)^{2}+k^{2}-k_{L}^{2}}-k} \frac{N_{\mathrm{f}}+1-k_{L}}{N_{\mathrm{f}}+1+k_{L}}\right)^{-\left(N_{\mathrm{f}}+1\right) k_{L}} \\
& \times\left(2 \frac{\left(N_{\mathrm{f}}+1\right) \sqrt{\left(N_{\mathrm{f}}+1\right)^{2}+k^{2}-k_{L}^{2}}-k k_{L}}{\left(N_{\mathrm{f}}+1\right)^{2}-k_{L}^{2}}\right)^{\frac{1}{2}-\left(\left(N_{\mathrm{f}}+1\right)^{2}+k_{L}^{2}\right)}
\end{aligned}
$$

The degeneracy of the saddle points is $\left(2 N_{\mathrm{f}}+2\right) ! /\left[\left(N_{\mathrm{f}}+1+k_{L}\right) !\left(N_{\mathrm{f}}+1-k_{L}\right) !\right]$. Combining all contributions we arrive at the main result of this subsection,

$$
\begin{aligned}
& Y_{N_{\mathrm{f}}} \stackrel{N \gg 1}{\approx} \frac{(-1)^{\left(N_{\mathrm{f}}+k_{L}+1\right)(N-1)} 2^{(N-2) / 2} \pi^{(N-1)^{2} / 2} \mathrm{e}^{\frac{N}{2}\left(k_{L}\left(\lambda_{-}^{2}-\lambda_{+}^{2}\right)-\left(N_{\mathrm{f}}+1\right)\left(\lambda_{+}^{2}+\lambda_{-}^{2}-4\right)\right)}}{N^{\frac{(N-1)^{2}}{2}+k_{L}^{2}-\left(N_{\mathrm{f}}+1\right)^{2}-\frac{1}{2}}\left(\left(N_{\mathrm{f}}+1\right)^{2}+k^{2}-k_{L}^{2}\right)^{1 / 4}} \\
& \times \frac{\left(\prod_{j=0}^{N_{\mathrm{f}}+k_{L}} j !\right)\left(\prod_{j=0}^{N_{\mathrm{f}}-k_{L}} j !\right)}{\prod_{j=0}^{2 N_{\mathrm{f}}+1} j !} \\
& \times\left(\frac{\sqrt{\left(N_{\mathrm{f}}+1\right)^{2}+k^{2}-k_{L}^{2}}+k}{\sqrt{\left(N_{\mathrm{f}}+1\right)^{2}+k^{2}-k_{L}^{2}}-k} \frac{N_{\mathrm{f}}+1-k_{L}}{N_{\mathrm{f}}+1+k_{L}}\right)^{k_{L}\left(N-N_{\mathrm{f}}-1\right)} \\
& \times\left(2 \frac{\left(N_{\mathrm{f}}+1\right) \sqrt{\left(N_{\mathrm{f}}+1\right)^{2}+k^{2}-k_{L}^{2}}-k k_{L}}{\left(N_{\mathrm{f}}+1\right)^{2}-k_{L}^{2}}\right)^{\left(N_{\mathrm{f}}+1\right)^{2}-3 k_{L}^{2}+\frac{1}{2}} \\
& \times \int \mathrm{d} \mu(U) \mathrm{e}^{-\operatorname{Tr} U^{\dagger} \operatorname{diag}\left(\lambda_{-} \mathbb{1}_{N_{\mathrm{f}}+1+k_{L}}, \lambda_{+} \mathbb{1}_{N_{\mathrm{f}}+1-k_{L}}\right) U \widehat{\widetilde{M}}} .
\end{aligned}
$$

and for integer $k$, implying $k=k_{L}$ and $\lambda_{ \pm}= \pm 1$, it reduces to

$$
\begin{aligned}
& Y_{N_{\mathrm{f}}} \stackrel{N \gg 1}{\approx} \frac{(-1)^{\left(N_{\mathrm{f}}+k+1\right)(N-1)} 2^{\frac{N-1}{2}+\left(N_{\mathrm{f}}+1\right)^{2}-3 k^{2}} \pi^{(N-1)^{2} / 2} \mathrm{e}^{N\left(N_{\mathrm{f}}+1\right)}}{N^{\frac{(N-1)^{2}}{2}+k^{2}-\left(N_{\mathrm{f}}+1\right)^{2}-\frac{1}{2}} \sqrt{N_{\mathrm{f}}+1}} \frac{\left(\prod_{j=0}^{N_{\mathrm{f}}+k} j !\right)\left(\prod_{j=0}^{N_{\mathrm{f}}-k} j !\right)}{\prod_{j=0}^{2 N_{\mathrm{f}}+1} j !} \\
& \times \int \mathrm{d} \mu(U) \mathrm{e}^{\operatorname{Tr} U^{\dagger} \operatorname{diag}\left(\mathbb{1}_{N_{\mathrm{f}}+1+k},-\mathbb{1}_{N_{\mathrm{f}}+1-k}\right) U \widehat{\widetilde{M}}}
\end{aligned}
$$

Open Access. This article is distributed under the terms of the Creative Commons Attribution License (CC-BY 4.0), which permits any use, distribution and reproduction in any medium, provided the original author(s) and source are credited. 


\section{References}

[1] H. Leutwyler and A.V. Smilga, Spectrum of Dirac operator and role of winding number in QCD, Phys. Rev. D 46 (1992) 5607 [inSPIRE].

[2] E.V. Shuryak and J.J.M. Verbaarschot, Random matrix theory and spectral sum rules for the Dirac operator in QCD, Nucl. Phys. A 560 (1993) 306 [hep-th/9212088] [INSPIRE].

[3] J.J.M. Verbaarschot and I. Zahed, Spectral density of the QCD Dirac operator near zero virtuality, Phys. Rev. Lett. 70 (1993) 3852 [hep-th/9303012] [INSPIRE].

[4] J.J.M. Verbaarschot, The spectrum of the QCD Dirac operator and chiral random matrix theory: the threefold way, Phys. Rev. Lett. 72 (1994) 2531 [hep-th/9401059] [InSPIRE].

[5] J.J.M. Verbaarschot, Universal behavior in Dirac spectra, in Confinement, duality and nonperturbative aspects of QCD. Proceedings, NATO Advanced Study Institute, Newton Institute Workshop, Cambridge, U.K., 23 June-4 July 1997, pg. 343 [hep-th/9710114] [INSPIRE].

[6] J.J.M. Verbaarschot and T. Wettig, Random matrix theory and chiral symmetry in $Q C D$, Ann. Rev. Nucl. Part. Sci. 50 (2000) 343 [hep-ph/0003017] [InSPIRE].

[7] J.J.M. Verbaarschot, QCD, chiral random matrix theory and integrability, in Application of random matrices in physics. Proceedings, NATO Advanced Study Institute, Les Houches, France, 6-25 June 2004, pg. 163 [hep-th/0502029] [INSPIRE].

[8] T. Kanazawa, Dirac spectra in dense QCD, Springer theses 124, Springer, Japan (2013).

[9] G. Akemann, Random matrix theory and quantum chromodynamics, Les Houches lecture notes, Oxford University Press, Oxford, U.K. (2016) [arXiv:1603.06011] [InSPIRE].

[10] R.D. Pisarski, Chiral symmetry breaking in three-dimensional electrodynamics, Phys. Rev. D 29 (1984) 2423 [INSPIRE].

[11] T.W. Appelquist, M.J. Bowick, D. Karabali and L.C.R. Wijewardhana, Spontaneous chiral symmetry breaking in three-dimensional QED, Phys. Rev. D 33 (1986) 3704 [INSPIRE].

[12] T. Appelquist, M.J. Bowick, D. Karabali and L.C.R. Wijewardhana, Spontaneous breaking of parity in $(2+1)$-dimensional QED, Phys. Rev. D 33 (1986) 3774 [INSPIRE].

[13] P.A. Lee, N. Nagaosa and X.-G. Wen, Doping a Mott insulator: physics of high-temperature superconductivity, Rev. Mod. Phys. 78 (2006) 17 [InSPIRE].

[14] C. Nayak, S.H. Simon, A. Stern, M. Freedman and S. Das Sarma, Non-Abelian anyons and topological quantum computation, Rev. Mod. Phys. 80 (2008) 1083 [arXiv:0707.1889] [INSPIRE].

[15] L. Balents, Spin liquids in frustrated magnets, Nature 464 (2010) 199.

[16] X.L. Qi and S.C. Zhang, Topological insulators and superconductors, Rev. Mod. Phys. 83 (2011) 1057 [arXiv: 1008.2026] [INSPIRE].

[17] T. Hansson, M. Hermanns, S. Simon and S. Viefers, Quantum Hall physics: hierarchies and conformal field theory techniques, Rev. Mod. Phys. 89 (2017) 025005.

[18] D.K. Hong and S.H. Park, Dynamical mass generation in $(2+1)$-dimensional QED with a Chern-Simons term, Phys. Rev. D 47 (1993) 3651 [InSPIRE].

[19] K.I. Kondo and P. Maris, First-order phase transition in three-dimensional QED with Chern-Simons term, Phys. Rev. Lett. 74 (1995) 18 [hep-ph/9408210] [INSPIRE].

[20] D.K. Hong, Zero temperature chiral phase transition in $(2+1)$-dimensional QED with a Chern-Simons term, Phys. Rev. D 57 (1998) 1313 [hep-th/9708027] [INSPIRE]. 
[21] T. Itoh and H. Kato, Dynamical generation of fermion mass and magnetic field in three-dimensional QED with Chern-Simons term, Phys. Rev. Lett. 81 (1998) 30 [hep-th/9802101] [INSPIRE].

[22] T. Matsuyama, H. Nagahiro and S. Uchida, A dynamical mass generation of a two component fermion in the Maxwell-Chern-Simons $Q E D_{3}$ : the lowest ladder approximation, Phys. Rev. D 60 (1999) 105020 [hep-th/9901049] [INSPIRE].

[23] G.-Z. Liu and G. Cheng, Effect of gauge boson mass on chiral symmetry breaking in $Q E D_{3}$, Phys. Rev. D 67 (2003) 065010 [hep-th/0211231] [InSPIRE].

[24] C.P. Hofmann, A. Raya and S.S. Madrigal, Confinement in Maxwell-Chern-Simons planar quantum electrodynamics and the $1 / N$ approximation, Phys. Rev. D 82 (2010) 096011 [arXiv: 1010.3466] [INSPIRE].

[25] N. Karthik and R. Narayanan, No evidence for bilinear condensate in parity-invariant three-dimensional QED with massless fermions, Phys. Rev. D 93 (2016) 045020 [arXiv: 1512.02993] [INSPIRE].

[26] N. Karthik and R. Narayanan, Scale-invariance of parity-invariant three-dimensional QED, Phys. Rev. D 94 (2016) 065026 [arXiv:1606.04109] [INSPIRE].

[27] N. Karthik and R. Narayanan, Flavor and topological current correlators in parity-invariant three-dimensional QED, Phys. Rev. D 96 (2017) 054509 [arXiv: 1705.11143] [INSPIRE].

[28] D. Roscher, E. Torres and P. Strack, Dual $Q E D_{3}$ at " $N_{F}=1 / 2$ " is an interacting CFT in the infrared, JHEP 11 (2016) 017 [arXiv:1605.05347] [INSPIRE].

[29] N. Karthik and R. Narayanan, Parity anomaly cancellation in three-dimensional QED with a single massless Dirac fermion, Phys. Rev. Lett. 121 (2018) 041602 [arXiv:1803.03596] [inSPIRE].

[30] C. Vafa and E. Witten, Restrictions on symmetry breaking in vector-like gauge theories, Nucl. Phys. B 234 (1984) 173 [inSPIRE].

[31] T. Appelquist and D. Nash, Critical behavior in $(2+1)$-dimensional QCD, Phys. Rev. Lett. 64 (1990) 721 [INSPIRE].

[32] G. Ferretti, S.G. Rajeev and Z. Yang, The effective Lagrangian of three-dimensional quantum chromodynamics, Int. J. Mod. Phys. A 7 (1992) 7989 [hep-th/9204075] [INSPIRE].

[33] M.C. Diamantini, P. Sodano and G.W. Semenoff, Chiral dynamics and fermion mass generation in three-dimensional gauge theory, Phys. Rev. Lett. 70 (1993) 3848 [hep-ph/9301256] [INSPIRE].

[34] P.H. Damgaard, U.M. Heller, A. Krasnitz and T. Madsen, A quark-anti-quark condensate in three-dimensional QCD, Phys. Lett. B 440 (1998) 129 [hep-lat/9803012] [INSPIRE].

[35] N. Karthik and R. Narayanan, Bilinear condensate in three-dimensional large- $N_{c} Q C D$, Phys. Rev. D 94 (2016) 045020 [arXiv: 1607.03905] [INSPIRE].

[36] J.J.M. Verbaarschot and I. Zahed, Random matrix theory and QCD in three-dimensions, Phys. Rev. Lett. 73 (1994) 2288 [hep-th/9405005] [INSPIRE].

[37] T. Nagao and K. Slevin, Nonuniversal correlations for random matrix ensembles, J. Math. Phys. 34 (1993) 2075.

[38] P.H. Damgaard and S.M. Nishigaki, Universal massive spectral correlators and QCD in three-dimensions, Phys. Rev. D 57 (1998) 5299 [hep-th/9711096] [INSPIRE].

[39] G. Akemann and P.H. Damgaard, Microscopic spectra of Dirac operators and finite volume partition functions, Nucl. Phys. B 528 (1998) 411 [hep-th/9801133] [INSPIRE]. 
[40] J. Christiansen, Odd flavored $Q C D_{3}$ and random matrix theory, Nucl. Phys. B 547 (1999) 329 [hep-th/9809194] [INSPIRE].

[41] U. Magnea, The orthogonal ensemble of random matrices and QCD in three-dimensions, Phys. Rev. D 61 (2000) 056005 [hep-th/9907096] [INSPIRE].

[42] U. Magnea, Three-dimensional QCD in the adjoint representation and random matrix theory, Phys. Rev. D 62 (2000) 016005 [hep-th/9912207] [INSPIRE].

[43] C. Hilmoine and R. Niclasen, The microscopic spectral density of the Dirac operator derived from Gaussian orthogonal and symplectic ensembles, Phys. Rev. D 62 (2000) 096013 [hep-th/0004081] [INSPIRE].

[44] T. Nagao and S.M. Nishigaki, Massive random matrix ensembles at $\beta=1$ and $\beta=4: Q C D$ in three-dimensions, Phys. Rev. D 63 (2001) 045011 [hep-th/0005077] [INSPIRE].

[45] R.J. Szabo, Microscopic spectrum of the QCD Dirac operator in three dimensions, Nucl. Phys. B 598 (2001) 309 [hep-th/0009237] [INSPIRE].

[46] R.J. Szabo, Finite volume gauge theory partition functions in three dimensions, Nucl. Phys. B 723 (2005) 163 [hep-th/0504202] [InSPIRE].

[47] S. Deser, R. Jackiw and S. Templeton, Topologically massive gauge theories, Annals Phys. 140 (1982) 372 [Erratum ibid. 185 (1988) 406] [Annals Phys. 281 (2000) 409] [INSPIRE].

[48] Z. Komargodski and N. Seiberg, A symmetry breaking scenario for QCD $D_{3}$, JHEP 01 (2018) 109 [arXiv: 1706.08755] [INSPIRE].

[49] D. Gaiotto, Z. Komargodski and N. Seiberg, Time-reversal breaking in $Q C D_{4}$, walls and dualities in $2+1$ dimensions, JHEP 01 (2018) 110 [arXiv:1708.06806] [INSPIRE].

[50] A. Armoni and V. Niarchos, Phases of $Q C D_{3}$ from non-SUSY Seiberg duality and brane dynamics, Phys. Rev. D 97 (2018) 106001 [arXiv:1711.04832] [InSPIRE].

[51] M. Mariño, Chern-Simons theory, matrix integrals and perturbative three manifold invariants, Commun. Math. Phys. 253 (2004) 25 [hep-th/0207096] [INSPIRE].

[52] M. Aganagic, A. Klemm, M. Mariño and C. Vafa, Matrix model as a mirror of Chern-Simons theory, JHEP 02 (2004) 010 [hep-th/0211098] [INSPIRE].

[53] M. Tierz, Soft matrix models and Chern-Simons partition functions, Mod. Phys. Lett. A 19 (2004) 1365 [hep-th/0212128] [INSPIRE].

[54] A. Kapustin, B. Willett and I. Yaakov, Exact results for Wilson loops in superconformal Chern-Simons theories with matter, JHEP 03 (2010) 089 [arXiv:0909.4559] [INSPIRE].

[55] N. Drukker, M. Mariño and P. Putrov, From weak to strong coupling in ABJM theory, Commun. Math. Phys. 306 (2011) 511 [arXiv:1007.3837] [inSPIRE].

[56] A. Armoni and V. Niarchos, $Q C D_{3}$ with two-index quarks, mirror symmetry and fivebrane anti-BIons near orientifolds, Phys. Rev. D 98 (2018) 114009 [arXiv:1808.07715] [INSPIRE].

[57] S.R. Das, A. Dhar, A.M. Sengupta and S.R. Wadia, New critical behavior in $d=0$ large $N$ matrix models, Mod. Phys. Lett. A 5 (1990) 1041 [INSPIRE].

[58] G.M. Cicuta and E. Montaldi, Matrix models and marginal operators in the planar limit, Mod. Phys. Lett. A 5 (1990) 1927 [INSPIRE].

[59] H. Ueda, Regularization of quantum gravity in the matrix model approach, Prog. Theor. Phys. 86 (1991) 23 [INSPIRE]. 
[60] S. Sawada and H. Ueda, Nonperturbative effect of a modified action in matrix models, Mod. Phys. Lett. A 6 (1991) 3717 [InSPIRE].

[61] G.P. Korchemsky, Matrix model perturbed by higher order curvature terms, Mod. Phys. Lett. A 7 (1992) 3081 [hep-th/9205014] [INSPIRE].

[62] F. David, A scenario for the $c>1$ barrier in noncritical bosonic strings, Nucl. Phys. B 487 (1997) 633 [hep-th/9610037] [INSPIRE].

[63] M. Kieburg, J.J.M. Verbaarschot and S. Zafeiropoulos, Spectral properties of the Wilson Dirac operator and random matrix theory, Phys. Rev. D 88 (2013) 094502 [arXiv: 1307.7251] [INSPIRE].

[64] J. Gasser and H. Leutwyler, Thermodynamics of chiral symmetry, Phys. Lett. B 188 (1987) 477 [INSPIRE].

[65] L. Álvarez-Gaumé, S. Della Pietra and G.W. Moore, Anomalies and odd dimensions, Annals Phys. 163 (1985) 288 [INSPIRE].

[66] H. Leutwyler, Dirac operator and Chern-Simons action, Helv. Phys. Acta 63 (1990) 660 [INSPIRE].

[67] A.N. Redlich, Parity violation and gauge noninvariance of the effective gauge field action in three-dimensions, Phys. Rev. D 29 (1984) 2366 [INSPIRE].

[68] S. Deser, L. Griguolo and D. Seminara, Effective QED actions: representations, gauge invariance, anomalies and mass expansions, Phys. Rev. D 57 (1998) 7444 [hep-th/9712066] [INSPIRE].

[69] A.M. Halasz and J.J.M. Verbaarschot, Effective Lagrangians and chiral random matrix theory, Phys. Rev. D 52 (1995) 2563 [hep-th/9502096] [InSPIRE].

[70] A.C. Bertuola, O. Bohigas and M.P. Pato, Family of generalized random matrix ensembles, Phys. Rev. E 70 (2004) 065102 [math-ph/0411033].

[71] A.Y. Abul-Magd, Random matrix theory within superstatistics, Phys. Rev. E 72 (2005) 066114 [cond-mat/0510494].

[72] G. Akemann and P. Vivo, Power law deformation of Wishart-Laguerre ensembles of random matrices, J. Stat. Mech. 0809 (2008) P09002 [arXiv:0806.1861] [InSPIRE].

[73] T. Kanazawa, Heavy-tailed chiral random matrix theory, JHEP 05 (2016) 166 [arXiv: 1602.05631] [INSPIRE].

[74] R.D. Pisarski, Effective theory of Wilson lines and deconfinement, Phys. Rev. D 74 (2006) 121703 [hep-ph/0608242] [INSPIRE].

[75] J.C. Myers and M.C. Ogilvie, New phases of SU(3) and SU(4) at finite temperature, Phys. Rev. D 77 (2008) 125030 [arXiv:0707.1869] [INSPIRE].

[76] M. Ünsal and L.G. Yaffe, Center-stabilized Yang-Mills theory: confinement and large $N$ volume independence, Phys. Rev. D 78 (2008) 065035 [arXiv:0803.0344] [INSPIRE].

[77] G. Akemann, D. Dalmazi, P.H. Damgaard and J.J.M. Verbaarschot, $Q C D_{3}$ and the replica method, Nucl. Phys. B 601 (2001) 77 [hep-th/0011072] [INSPIRE].

[78] G. Akemann and G. Vernizzi, Characteristic polynomials of complex random matrix models, Nucl. Phys. B 660 (2003) 532 [hep-th/0212051] [INSPIRE]. 
[79] E. Strahov and Y.V. Fyodorov, Universal results for correlations of characteristic polynomials: Riemann-Hilbert approach, Commun. Math. Phys. 241 (2003) 343 [math-ph/0210010] [INSPIRE].

[80] K. Splittorff and J.J.M. Verbaarschot, Factorization of correlation functions and the replica limit of the Toda lattice equation, Nucl. Phys. B 683 (2004) 467 [hep-th/0310271] [INSPIRE].

[81] T. Kanazawa and M. Kieburg, Symmetry crossover protecting chirality in Dirac spectra, JHEP 11 (2018) 205 [arXiv: 1809.10602] [INSPIRE].

[82] Harish-Chandra, Invariant differential operators on a semisimple Lie algebra, Proc. Nat. Acad. Sci. 42 (1956) 252.

[83] C. Itzykson and J.B. Zuber, The planar approximation. 2, J. Math. Phys. 21 (1980) 411 [INSPIRE].

[84] K.A. Andréief, Note sur une relation les intégrales définies des produits des fonctions (in French), Mém. Soc. Sci. Bordeaux 2 (1883) 1.

[85] I.S. Gradshteyn and I.M. Ryzhik, Table of integrals, series and products, seventh ed., Elsevier, The Netherlands (2007).

[86] G. Akemann, J.C. Osborn, K. Splittorff and J.J.M. Verbaarschot, Unquenched QCD Dirac operator spectra at nonzero baryon chemical potential, Nucl. Phys. B 712 (2005) 287 [hep-th/0411030] [INSPIRE].

[87] J.C. Osborn, K. Splittorff and J.J.M. Verbaarschot, Chiral symmetry breaking and the Dirac spectrum at nonzero chemical potential, Phys. Rev. Lett. 94 (2005) 202001 [hep-th/0501210] [INSPIRE].

[88] G. Akemann, T. Kanazawa, M.J. Phillips and T. Wettig, Random matrix theory of unquenched two-colour QCD with nonzero chemical potential, JHEP 03 (2011) 066 [arXiv: 1012.4461] [INSPIRE].

[89] T. Kanazawa, T. Wettig and N. Yamamoto, Singular values of the Dirac operator in dense QCD-like theories, JHEP 12 (2011) 007 [arXiv:1110.5858] [INSPIRE].

[90] J.J.M. Verbaarschot and T. Wettig, Dirac spectrum of one-flavor QCD at $\theta=0$ and continuity of the chiral condensate, Phys. Rev. D 90 (2014) 116004 [arXiv:1407.8393] [INSPIRE].

[91] D. Dominici, Asymptotic analysis of the Hermite polynomials from their differential-difference equation, J. Diff. Eq. Appl. 13 (2007) 1115 [math.CA/0601078].

[92] M.E. Peskin, The alignment of the vacuum in theories of technicolor, Nucl. Phys. B 175 (1980) 197 [INSPIRE].

[93] A. Armoni, T.T. Dumitrescu, G. Festuccia and Z. Komargodski, Metastable vacua in large- $N$ $Q C D_{3}$, arXiv:1905.01797 [inSPIRE].

[94] H.-J. Sommers, Superbosonization, Acta Phys. Polon. B 38 (2007) 4105 [arXiv:0710.5375].

[95] P. Littelmann, H.J. Sommers and M.R. Zirnbauer, Superbosonization of invariant random matrix ensembles, Commun. Math. Phys. 283 (2008) 343 [arXiv:0707.2929].

[96] M. Kieburg, H.-J. Sommers and T. Guhr, A comparison of the superbosonization formula and the generalized Hubbard-Stratonovich transformation, J. Phys. A 42 (2009) 275206 [arXiv: 0905.3256]. 